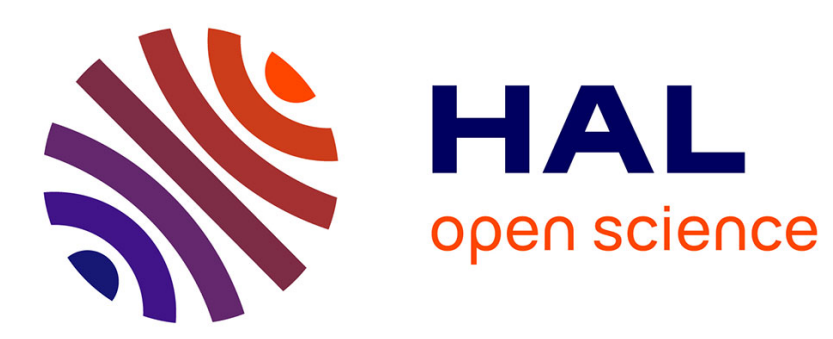

\title{
Consistent closure schemes for statistical models of anisotropic fluids
}

\author{
Martin Kröger, Amine Ammar, Francisco Chinesta
}

\section{To cite this version:}

Martin Kröger, Amine Ammar, Francisco Chinesta. Consistent closure schemes for statistical models of anisotropic fluids. Journal of Non-Newtonian Fluid Mechanics, 2008, 149 (1-3), pp.40 - 55. 10.1016/j.jnnfm.2007.05.007 . hal-01005990

\section{HAL Id: hal-01005990 https://hal.science/hal-01005990}

Submitted on 2 Apr 2017

HAL is a multi-disciplinary open access archive for the deposit and dissemination of scientific research documents, whether they are published or not. The documents may come from teaching and research institutions in France or abroad, or from public or private research centers.
L'archive ouverte pluridisciplinaire HAL, est destinée au dépôt et à la diffusion de documents scientifiques de niveau recherche, publiés ou non, émanant des établissements d'enseignement et de recherche français ou étrangers, des laboratoires publics ou privés. 


\title{
Consistent closure schemes for statistical models of anisotropic fluids
}

\author{
Martin Kröger ${ }^{\mathrm{a}}$, Amine Ammar ${ }^{\mathrm{b}}$, Francisco Chinesta ${ }^{\mathrm{c}}$ \\ ${ }^{a}$ Polymer Physics, ETH Zurich, Department of Materials, Wolfgang-Pauli-Str. 10, CH-8093 Zürich, Switzerland \\ ${ }^{\mathrm{b}}$ Laboratoire de Rhéologie, UMR 5520 CNRS-UJF-INPG, 1301 Rue de la Piscine, \\ BP 53 Domaine Universitaire, F-38041 Grenoble Cedex 9, France \\ ${ }^{\mathrm{c}}$ LMSP UMR 8106 CNRS-ENSAM-ESEM, 151 Boulevard de l'Hôpital, F-75013 Paris, France
}

\begin{abstract}
We propose a rational approach to approximating the various alignment tensors. It preserves the correct symmetry and leads to consistent results. For the case of uniaxial nematic fluids, the decoupling approximation for a tensor of rank $n$ involves $(n-2) / 2$ scalar functions $S_{n}\left(S_{2}\right)$ in terms of a scalar argument $S_{2}$, with $S_{n}(0)=0$ and $S_{n}(1)=1$. Nothing else can be concluded about the mathematical relationship between moments of the distribution function, and in particular, all consistent decoupling approximations for fourth-order moment in terms of second-order moments can be characterized by a single $S_{4}\left(S_{2}\right)$ function. We propose using the simple model dependent convex shaped equilibrium relationship between $S_{4}$ and $S_{2}$ to characterize new (and simple) decoupling approximations K-I and K-II for the biaxial (including uniaxial) phase. In order to test the new against earlier proposed approximations rigorously, and to discuss consistency issues, we solve the Hess-Doi Fokker-Planck equation for nematic and nematic-discotic liquid crystals efficiently for a wide range of (2300 distinct) possible conditions including mixed shear and elongational flows, diverse field strengths, and molecular shapes. As a result, we confirm the closures K-I and K-II with correct tensorial symmetry; they are valid under arbitrary conditions to high precision, exact in the isotropic and totally aligned phases, improve upon earlier parameter-free closures in particular in the temperature regime $T \in[0.6, \infty] \times T_{\mathrm{NI}}$ with the nematic-isotropic transition temperature $T_{\mathrm{NI}}$ (or alternatively, for mean-field strengths $U \in[0,8]$ ). K-II performs as good as the so-called Bingham closure, which usually requires 30 empirical coefficients, while K-I and KII are essentially parameter-free, and their quality can be expected to be insensitive to the particular model.
\end{abstract}

Keywords: Anisotropic fluids; Closure schemes; Nematic fluids; Alignment tensors; Ferrofluids; Numerical solution of Fokker-Planck equation

\section{Introduction}

Anisotropic fluids consist of particles or molecules that can be aligned by flow and external fields. One approach to modeling such fluids is to introduce a set of unit vectors fields, usually called directors, that represent the preferred directions of the particle orientations. If there are fluctuations in the particle orientations, however, the alignment of the particles is not perfect. In this case, the directors represent the particle orientations in an averaged, macroscopic sense. This director approach has been quite successful in modeling low molecular weight liquid crystals where the degree of alignment is generally constant [1-4], and in modelling ferrofluids [5-8].
For some anisotropic fluids, such as liquid crystal polymers, particle fluctuations play an important role in the overall properties. An alternative description that explicitly takes into account fluctuations in particle orientations and the resulting variable degree of alignment is a statistical one with a distribution function providing the information for the particle orientations. The orientation distribution function satisfies an evolution equation (for a review see, e.g., [9]). In general, however, this evolution equation can be solved only numerically. And for most microstructural models, such as the Hess-Doi model for rigid, rod-like liquid crystal polymers [10,11], the complete numerical solution of the orientation distribution function at present is quite prohibitive and impractical [12] for common applications, cf. [13] for solution methods. Approximative solutions are also available [14-16] which allow to characterize the spatiotemporal behavior of liquid crystals $[17,18]$.

Alternatively to solving directly for the distribution function, one can reformulate the statistical model in terms of a 
hierarchy of higher tensorial moments of the alignment (the socalled alignment tensors) and then solve the resulting evolution equations for the alignment tensors. The alignment tensors are useful since their principal directions are related to the macroscopic directors and their principal values are related to the scalar order parameters that characterize the variable degree of alignment. Since there is in general an infinite hierarchy of coupled evolution equations for the alignment tensors, this problem is also computationally difficult, so that various closure schemes have been introduced to relate higher moments of the alignment to lower moments. The most common closure schemes relate the fourth-order alignment tensor to the secondorder one. Such approximations simplify considerably the effort to obtain the macroscopic alignment, and a large amount of work has been invested in studying them. The proposed schemes include linear [19] and quadratic closure [10,20], interpolation between the limiting cases of weak alignment and perfect alignment [21], truncation of the evolution equations after a certain order [9,10,14-16,22,23], maximum entropy method [24], timestructure invariance criteria [25,26], and specification of an a priori form of the orientation distribution function [27-31].

These closure schemes have been proposed often on an ad hoc basis and are sometimes inconsistent with the exact equations based on the orientation distribution function [12,32,33]. For example, in the Doi and Edwards [20] model the quadratic closure gives an incorrect expression for the fourth-order alignment tensor in the isotropic phase as well as an incorrect orientation of the director in the uniaxial phase, while being still compatible with time-structure invariance criteria [25]. Some closure schemes also commonly lead to pathological results for certain parameter ranges [12,34]. One reason for this inconsistency is that the various schemes yield an overdetermined system of equations for the principal directions and principal values of the alignment tensors. In particular, any scheme that approximates both principal values and principal directions can lead to an overdetermined system.

The purpose of this paper is to show how this overdeterminacy arises and, most importantly, how to formulate consistent closure schemes so that it does not arise. This leads us to propose new and simple closure schemes independent of the particular microstructural model. Our procedure is based on the representation of the alignment tensors in terms of their principal values and principal directions. These representations show that only the independent principal values of the alignment tensors need to be approximated in a closure scheme. For example, specifying the principal values of the fourth-order alignment tensor in terms of the principal values of the second-order alignment tensor leads to a consistent second-order closure scheme. By avoiding any assumptions on the principal directions, our procedure maintains the correct symmetry and preferred orientations, thus leading to consistent, non-pathological results. Although our procedure is quite general, for simplicity we treat in detail only the second to fourth-order alignment tensors.

We begin with a review of measures of alignment for anisotropic fluids. The symmetry is conveniently divided into three cases according to the number of distinct principal values: isotropic, uniaxial and biaxial. We examine all three cases and discuss consistent closure schemes for each case. In particular, for the uniaxial case, a consistent closure scheme requires specifying only a single scalar parameter. For the biaxial case, three scalar parameters must be specified. Furthermore, the choice of these three relations is strongly restricted by the requirement that the fourth-order alignment tensor be expressible in terms of the second-order alignment tensor. We also show that a modified quadratic closure relation can hold for all three types of symmetry, which is important for materials that can exhibit all three types of symmetry, such as liquid crystal polymers in complex flows. Our results demonstrate, however, that most commonly used closure schemes are inconsistent and, hence, lead to incorrect results. An exception, although, is the scheme that postulates an a priori form for the orientation distribution function.

As an illustration of our procedure, we apply it to the Hess-Doi model for rigid, rod-like polymers and to ferrofluids. The results can be useful for simulating complex flows such as those arising in the injection molding of liquid crystalline polymers into high strength parts.

\section{Orientational distribution function}

For uniaxial-shaped particles with symmetry axis $\boldsymbol{u}$, the orientational (part of a eventually space and time-dependent) distribution function $f(\boldsymbol{u})$ with $u^{2}=1$ can be expanded in terms of Cartesian symmetric traceless (anisotropic, irreducible) tensors $\boldsymbol{u}_{[n]} \equiv \widetilde{\boldsymbol{u}_{(l)}}$ of rank $n$, with $\boldsymbol{u}_{(n)} \equiv \boldsymbol{u} \boldsymbol{u} \ldots \boldsymbol{u}$ the $n$-fold tensorial product of vector $\boldsymbol{u}$, the symbol $\ldots$ denoting the anisotropic part, and the tensorial coefficients in front of the $\boldsymbol{u}_{[n]}$ 's are determined by multiplying $f$ with $\boldsymbol{u}_{[n]}$ and subsequent integration over the unit sphere, to yield

$$
\begin{aligned}
f(\boldsymbol{u}) & =\frac{1}{4 \pi}\left(1+\sum_{n=1}^{\infty}\left\langle\zeta_{n} \boldsymbol{u}_{[n]}\right\rangle \odot^{n}\left(\zeta_{n} \boldsymbol{u}_{[n]}\right)\right) \\
& =\frac{1}{4 \pi} \sum_{n=0}^{\infty} \zeta_{n}^{2} \boldsymbol{a}_{(n)} \odot^{n} \boldsymbol{u}_{[n]},
\end{aligned}
$$

where $\odot^{l}$ denotes an $n$-fold contraction and $\boldsymbol{a}_{[l]} \equiv\left\langle\boldsymbol{u}_{[l]}\right\rangle$ the $n$ th rank alignment tensor. The constant $(4 \pi)^{-1}$ ensures proper normalization $\langle 1\rangle=1$, and the average $\langle\ldots\rangle$ is defined through $\langle\ldots\rangle \equiv \int \ldots f(\boldsymbol{u}) \mathrm{d}^{2} u$. The prefactor

$\zeta_{n}=\sqrt{\frac{(2 n+1) ! !}{n !}}$

with $k ! !=k(k-2)(k-4) \ldots$ is immediately derived using the identity [9]

$\frac{1}{4 \pi} \int \boldsymbol{u}_{[k]} \boldsymbol{u}_{[n]} \mathrm{d}^{2} u=\frac{n !}{(2 n+1) ! !} \delta_{k n} \boldsymbol{\Delta}^{(n)}$,

where $\boldsymbol{\Delta}^{(n)}$ is the isotropic tensor [9,35]- and projector - of rank $n$ with the feature $\boldsymbol{\Delta}^{(n)} \odot^{n} \boldsymbol{a}_{(n)}=\boldsymbol{a}_{[n]}$ and just $\boldsymbol{\Delta}^{(0)}=1$ is needed here to prove (2). Within the statistical approach to the dynamics of anisotropic fluids, the distribution function obeys a Fokker-Planck (FP) equation from which coupled equations of moments (including anisotropic moments-the alignment 
tensors) are obtained by integration, cf. $[9,14,36]$ for an introduction.

\section{Alignment tensors}

A (symmetric traceless) alignment tensor $\boldsymbol{a}_{[l]}$ of rank $l$ is always uniquely expressed in terms of symmetric orientation tensors $\boldsymbol{a}_{(k)} \equiv\left\langle\boldsymbol{u}_{(k)}\right\rangle$ with $k \leq l$ and unity tensor $\boldsymbol{I}$ which is obvious from the existence of the above-mentioned isotropic tensor $\boldsymbol{\Delta}^{(l)}$. There is a closed formula available for the projector $\boldsymbol{\Delta}^{(l)}$, cf. Eq. (10.14) of [9], but since we do not need the higher-order tensors in this manuscript, we mention, that the anisotropic tensors can be constructed manually by writing down an ansatz like $\boldsymbol{a}_{[2]}=c_{1} \boldsymbol{a}_{(2)}+c_{2} \boldsymbol{I}, \boldsymbol{a}_{[3]}=\boldsymbol{a}_{(3)}+\left\{\boldsymbol{a}_{(1)} \boldsymbol{I}\right\}_{\text {sym }}$, or $\boldsymbol{a}_{[4]}=c_{1} \boldsymbol{a}_{(4)}+c_{2}\left\{\boldsymbol{a}_{(2)} \boldsymbol{I}\right\}_{\text {sym }}+c_{3}\{\boldsymbol{I} \boldsymbol{I}\}_{\text {sym }}$ with unknown coefficients $c$ and taking the (more precisely, an arbitrary) trace on both sides, because $\operatorname{tr}\left(\boldsymbol{a}_{[\ldots]}\right)$ vanishes by definition. Here, $\boldsymbol{I}$ is the identity tensor. With $\{\cdot\}_{\text {sym }}$ we denote the symmetric, normalized part, as $\{\boldsymbol{X}\}_{\text {sym }}$ and for clarity, now in component notation,

$$
\begin{aligned}
\left\{X_{\mu \nu}\right\}_{\text {sym }} \equiv & \frac{1}{2}\left(X_{\mu \nu}+X_{\nu \mu}\right) \\
\left\{x_{\mu} Y_{\nu \kappa}\right\}_{\text {sym }} \equiv & \frac{1}{3}\left(x_{\mu} Y_{\nu \kappa}+x_{\nu} Y_{\mu \kappa}+x_{\kappa} Y_{\mu \nu}\right) \\
\left\{X_{\mu \nu} Y_{\kappa \lambda}\right\}_{\text {sym }} \equiv & \frac{1}{6}\left(X_{\mu \nu} Y_{\kappa \lambda}+X_{\mu \kappa} Y_{\nu \lambda}+X_{\mu \lambda} Y_{\kappa \lambda}+X_{\nu \kappa} Y_{\mu \lambda}\right. \\
& \left.+X_{\nu \lambda} Y_{\mu \kappa}+X_{\kappa \lambda} Y_{\mu \nu}\right)
\end{aligned}
$$

for any symmetric 2nd rank tensors $\boldsymbol{X}, \boldsymbol{Y}$ and vector $\boldsymbol{x}$, the denominator equals the number of distinct terms. Accordingly, we obtain the following identities

$$
\begin{aligned}
& \boldsymbol{a}_{[2]}=\boldsymbol{a}_{(2)}-\frac{1}{3} \boldsymbol{I}, \\
& \boldsymbol{a}_{[3]}=\boldsymbol{a}_{(3)}-\frac{3}{5}\left\{\boldsymbol{a}_{(1)} \boldsymbol{I}\right\}_{\mathrm{sym}}, \\
& \boldsymbol{a}_{[4]}=\boldsymbol{a}_{(4)}-\frac{6}{7}\left\{\boldsymbol{a}_{(2)} \boldsymbol{I}\right\}_{\mathrm{sym}}+\frac{3}{35}\{\boldsymbol{I} \boldsymbol{I}\}_{\mathrm{sym}} .
\end{aligned}
$$

Odd moments are of relevance for polar materials such as dipolar fluids. For materials characterized through a director (in the presence of head-tail symmetry of molecules) only the even moments do not vanish. For example, the Eq. (7) shows that a relation for either $\boldsymbol{a}_{(4)}$ or $\boldsymbol{a}_{[4]}$ in terms of the second-order moment yields a corresponding relation for the other fourthorder alignment tensor. Obviously, $\operatorname{tr}\left(\boldsymbol{a}_{(1)}\right)=\boldsymbol{a}_{(1-2)}$ is consistent with (7) since $\operatorname{tr}\left(\boldsymbol{a}_{[\ldots]}\right)=0$. Notice, that (5)-(7) are also valid if one formally replaces $\boldsymbol{a}$ by $\boldsymbol{u}$ or $\boldsymbol{a}$ by an arbitrary unit vector $\boldsymbol{n}$.

Importantly, there are orthogonal unit vectors $\boldsymbol{n}, \boldsymbol{m}$ and $\boldsymbol{l}$ such that

$$
\begin{aligned}
& \boldsymbol{a}_{(2)}=\lambda_{1} \boldsymbol{n}_{(2)}+\lambda_{2} \boldsymbol{m}_{(2)}+\lambda_{3} \boldsymbol{l}_{(2)}, \\
& \boldsymbol{a}_{[2]}=\left(\lambda_{1}-\frac{1}{3}\right) \boldsymbol{n}_{(2)}+\left(\lambda_{2}-\frac{1}{3}\right) \boldsymbol{m}_{(2)}+\left(\lambda_{3}-\frac{1}{3}\right) \boldsymbol{l}_{(2)},
\end{aligned}
$$

where $\boldsymbol{n}_{(2)}=\boldsymbol{n} \boldsymbol{n}$ and $\boldsymbol{a}_{(2)}=\langle\boldsymbol{u} \boldsymbol{u}\rangle$, etc. by carrying over the notation introduced above. The $\lambda_{i}$ are the principal values of $\boldsymbol{a}_{(2)}$, and the unit vectors $\boldsymbol{n}, \boldsymbol{m}$ and $\boldsymbol{l}$ are the principal directions. The $\lambda_{i}$ are also constrained by the requirement that $\operatorname{tr}\left(\boldsymbol{a}_{(2)}\right)=1$ or, equivalently, $\operatorname{tr}\left(\boldsymbol{a}_{[2]}\right)=0$, thus $\sum_{i} \lambda_{i}=1$. Further, the identity $\boldsymbol{n}_{(2)}+\boldsymbol{m}_{(2)}+\boldsymbol{l}_{(2)}=\mathbf{0}$ can be used to eliminate, say, $\boldsymbol{l}_{(2)}$ from (8). Similar relations hold for alignment tensors of all orders, but for simplicity, we do not write them. The symmetry is directly related to the number of distinct principal values of the alignment tensors. For example, for the second-order moment $\boldsymbol{a}_{(2)}$, we have isotropic - 1 distinct principal value, uniaxial -2 distinct principal values biaxial -3 distinct principal values.

In the case of isotropy, the three principal values are all equal, $\lambda_{1}=\lambda_{2}=\lambda_{3}=\frac{1}{3}$, so that the second-order alignment tensors become

$\boldsymbol{a}_{(2)}=\frac{1}{3}\left(\boldsymbol{n}_{(2)}+\boldsymbol{m}_{(2)}+\boldsymbol{l}_{(2)}\right)=\frac{1}{3} \boldsymbol{I}, \quad \boldsymbol{a}_{[2]}=\mathbf{0}$.

Additionally, the third-order and fourth-order ones are $\boldsymbol{a}_{(3)}=$ $\boldsymbol{a}_{[3]}=\mathbf{0}$, and

$\boldsymbol{a}_{(4)}=\frac{1}{5}\{\boldsymbol{I I}\}_{\mathrm{sym}}, \quad \boldsymbol{a}_{[4]}=\mathbf{0}$.

It follows trivially from (9) and (10) that, in the isotropic phase,

$\boldsymbol{a}_{(4)}=\frac{9}{5}\left\{\boldsymbol{a}_{(2)} \boldsymbol{a}_{(2)}\right\}_{\mathrm{sym}}, \quad \boldsymbol{a}_{[4]}=\widehat{\boldsymbol{a}_{[2]} \boldsymbol{a}_{[2]}}$.

Thus, any closure relation for $\boldsymbol{a}_{(4)}$ must reduce to (11) in the isotropic phase. Importantly, this result shows that $\boldsymbol{a}_{(4)} \neq$ $\boldsymbol{a}_{(2)} \boldsymbol{a}_{(2)}$ in the isotropic case. The isotropic phase is a special case of the more general uniaxial and biaxial phases. We consider each case separately. Readers interested in the application of the new closures can skip the following Section 4; the more general expressions will be presented in Section 5.

\section{Uniaxial phase}

Scalar order parameters $S_{n}$ for the uniaxial phase are commonly defined through $S_{n} \equiv\left\langle P_{n}(\boldsymbol{u} \cdot \boldsymbol{n})\right\rangle$ with Legendre polynomial $P_{n}$, or equivalently, through $\boldsymbol{a}_{[n]}=S_{n} \boldsymbol{n}_{[n]}$. Inserting $\boldsymbol{a}_{[n]}$ into (1), one recovers the uniaxial orientational distribution function parameterized by order parameters and director $\boldsymbol{n}$. The isotropic phase is recovered for $\forall_{n} S_{n}=0$, the totally aligned uniaxial phase has $\forall_{n} S_{n}=1$. The squared order parameter $S_{n}$ can be calculated from the $n$th rank alignment tensors,

$S_{n}^{2}=\frac{(2 n-1) ! !}{n !} \boldsymbol{a}_{[n]} \odot^{n} \boldsymbol{a}_{[n]}$,

which can be also written as $S_{n}=\frac{(2 n-1) ! !}{n !} \boldsymbol{a}_{[n]} \odot^{n} \boldsymbol{n}_{[n]}$. Special cases of interest in the following discussion are $S_{1}=\langle\boldsymbol{u}\rangle^{2}$, and

$S_{2}^{2}=\frac{3}{2} \boldsymbol{a}_{[2]}: \boldsymbol{a}_{[2]}=\frac{1}{2}\left(3 \boldsymbol{a}_{(2)}: \boldsymbol{a}_{(2)}-1\right)$,

In the case of uniaxial symmetry, two of the principal values of the second-order alignment tensor are equal ( say $\lambda_{2}=\lambda_{3}$ ). 
In this case, the representation (8) has the simple form

$\boldsymbol{a}_{(2)}=S_{2} \boldsymbol{n}_{(2)}+\frac{1}{3}\left(1-S_{2}\right) \boldsymbol{I}, \quad \boldsymbol{a}_{[2]}=S_{2} \boldsymbol{n}_{[2]}$,

where $S_{2} \equiv\left(3 \lambda_{1}-1\right) / 2$. By making use of (6) and (7), the thirdand fourth-order moments are given by

$\boldsymbol{a}_{(3)}=S_{3} \boldsymbol{n}_{(3)}+\frac{3}{5}\left(S_{1}-S_{3}\right)\{\boldsymbol{I n}\}_{\mathrm{sym}}$,

$\boldsymbol{a}_{[3]}=S_{3} \boldsymbol{n}_{[3]}$,

$\boldsymbol{a}_{(4)}=S_{4} \boldsymbol{n}_{(4)}+\frac{6}{7}\left(S_{2}-S_{4}\right)\left\{\boldsymbol{I n}_{(2)}\right\}_{\mathrm{sym}}+\frac{1}{35}\left(7-10 S_{2}+3 S_{4}\right)\{\boldsymbol{I I}\}_{\mathrm{sym}}$,

$\boldsymbol{a}_{[4]}=S_{4} \boldsymbol{n}_{[4]}$.

The scalar order parameters $S_{2}, S_{3}$, and $S_{4}$ range in value by $0 \leq S_{2} \leq 1,-1 \leq S_{3} \leq 1,-\frac{3}{7} \leq S_{4} \leq 1$ which follows from $P_{2}(x)=\frac{1}{2}\left(3 x^{2}-1\right), P_{3}(x)=\frac{1}{2}\left(5 x^{3}-3 x\right)$, and $P_{4}(x)=\frac{1}{8}(3-$ $\left.30 x^{2}+35 x^{4}\right)$, respectively. The principal direction $\boldsymbol{n}$ is the uniaxial director. As in the isotropic case, the traceless moments $\boldsymbol{a}_{[\ldots]}$ have simpler forms than $\boldsymbol{a}_{(\ldots)}$. Notice that, due to (14), for the uniaxial case it is possible to solve explicitly for $\boldsymbol{n}_{(2)}$ in terms of $\boldsymbol{a}_{(2)}$ or $\boldsymbol{a}_{[2]}$.

\subsection{Closure for uniaxial phase}

Combining (14) with (15) yields a consistent closure relationship between 4 th and 2 nd moment in terms of a single scalar function $S_{4}\left(S_{2}\right)$ subject to the (important) constraints $S_{4}(0)=0$, $S_{4}(1)=1$, and $-\frac{3}{7} \leq S_{4} \leq 1$ for all $0 \leq S_{2} \leq 1$. It reads:

$\boldsymbol{a}_{(4)}=\alpha\left\{\boldsymbol{a}_{(2)} \boldsymbol{a}_{(2)}\right\}_{\mathrm{sym}}-2 \beta\left\{\boldsymbol{a}_{(2)} \boldsymbol{I}\right\}_{\mathrm{sym}}-2 \gamma\{\boldsymbol{I} \boldsymbol{I}\}_{\mathrm{sym}}$,

where

$\alpha \equiv \frac{S_{4}}{S_{2}^{2}}$,

$\beta \equiv \frac{\alpha}{3}+\frac{2 S_{4}}{21 S_{2}}-\frac{3}{7}$

$\gamma \equiv \frac{3}{70}+\frac{2 S_{4}}{45}-\frac{\alpha}{18}-\frac{2 S_{4}}{63 S_{2}}$.

or equivalently, in terms of anisotropic moments,

$\boldsymbol { a } _ { [ 4 ] } = \frac { S _ { 4 } } { S _ { 2 } ^ { 2 } } \longdiv { \boldsymbol { a } _ { [ 2 ] } \boldsymbol { a } _ { [ 2 ] } }$.

The extension for higher order moments reads

$\forall_{n, m} S_{n} S_{m} \boldsymbol{a}_{[n+m]}=S_{n+m} \overline{\boldsymbol{a}_{[n]} \boldsymbol{a}_{[m]}}$,

and we should also mention the equation of change for order parameters [9]:

$S_{n} \dot{S}_{n}=\frac{1}{2} \frac{\mathrm{d}}{\mathrm{d} t}\left(S_{n}^{2}\right)=\frac{(2 n-1) ! !}{n !} \boldsymbol{a}_{[n]} \odot^{n} \frac{\partial}{\partial t} \boldsymbol{a}_{[n]}$.

The order parameter $S_{2}$ in terms of $\boldsymbol{a}_{(2)}$, cf. (13), supplements the closure (16), (17). No assumption has been made other than uniaxial symmetry, so that these results are exact for uniaxial symmetry. Any valid closure should be characterized by a correctly bounded, scalar function $S_{4}\left(S_{2}\right)$. Eq. (17) or (18) reduces to (11)- the isotropic phase - for $S_{4}=0$, and to the totally aligned phase, $\boldsymbol{a}_{(4)}=\boldsymbol{n}_{(4)}$, for $S_{4}=S_{2}=1$, and it obeys $\operatorname{tr}\left(\boldsymbol{a}_{(4)}\right)=\boldsymbol{a}_{(2)}$.

First of all for convenience, let us write the scalar function in the following form

$S_{4}=S_{4}\left(S_{2}\right)=S_{2}\left[1-\left(1-S_{2}\right)^{\nu}\right]$

with an unspecified, positive exponent $v>0$, which may weakly depend on $S_{2}$. Experiments and simulation studies show, that $v$ lies in the range $v \in[0.3,0.8][9,22]$. Let us further motivate the representation (21) by stating a few examples. (i) The potential for a magnetic moment with orientation $\boldsymbol{u}$ in a dimensionless magnetic field $\boldsymbol{h}$ [14] and strength $h \equiv|\boldsymbol{h}|$ (Langevin parameter $h)$ is given by $V=-k_{\mathrm{B}} T \boldsymbol{h} \cdot \boldsymbol{u}$. The corresponding equilibrium distribution function reads $f(\boldsymbol{u})=\frac{h}{4 \pi \sinh (h)} \mathrm{e}^{\boldsymbol{h} \cdot \boldsymbol{u}}$ and the order parameters obey $S_{0}(h)=1, S_{1}(h)=\operatorname{coth}(h)-1 / h$, and for $i \geq 1$ :

$S_{i+1}(h)=-(2 i+1) h^{-1} S_{i}(h)+S_{i-1}(h)$,

from which follows, that $v \approx \frac{1}{2}$ for a ferrofluid close to equilibrium, for all $h$. (ii) More generally, a maximum entropy argument for a Maier-Saupe type potential yields $v \approx 3 / 5$ as can be seen as follows. For an orientational distribution function $f(\mathbf{u})=Z^{-1} \exp ^{a P_{2}(\boldsymbol{u} \cdot \boldsymbol{n})}$ (a uniaxial Bingham-type distribution) the scalar order parameters $S_{2}$ and $S_{4}$ are parameterized through $a$ as follows: $S_{2}=Z^{-1} \mathrm{~d} Z / \mathrm{d} a$ and $S_{4}=\frac{35}{18}\left(Z^{-1} \frac{\mathrm{d}^{2} Z}{\mathrm{~d} a^{2}}-\frac{1}{5}\right)-$ $\frac{5}{9} S_{2}$, where the partition function $Z$ is $Z=\int f(\boldsymbol{u}) d^{2} u$. Here, the resulting $S_{4}$ is always non-negative, and $v$ is well approximated by $3 / 5$, cf. Fig. 1 . We may call the closure (18) with $S_{4}\left(S_{2}\right)$ from $(21)$ and $v=3 / 5$ the uniaxial K-I closure which is a special case of the more general biaxial K-I closure.

Since closure relationships are needed to approximate a higher-order tensor in terms of lower-order ones, they are not used in their pure form (16) but in connection with a matrix contraction to lower rank. In particular, let $\boldsymbol{M}$ be an arbitrary, not necessarily symmetric, 2nd rank tensor. Inserting (21) into

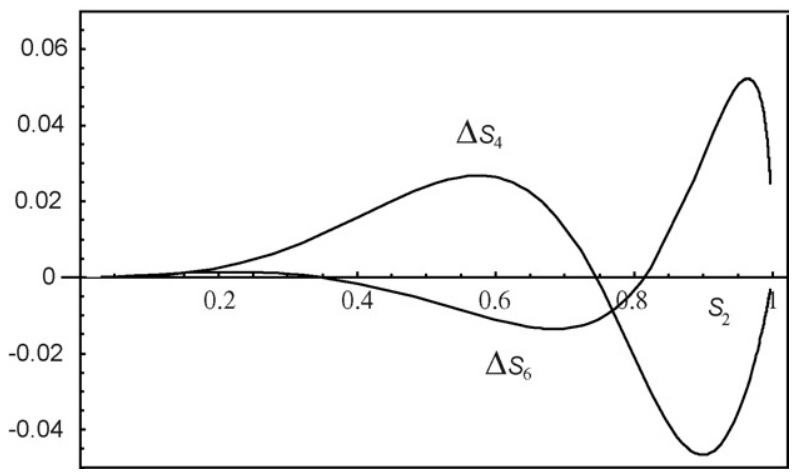

Fig. 1. Absolute quality of simple closure relationships $S_{4}\left(S_{2}\right)=S_{2}(1-$ $\left.\left(1-S_{2}\right)^{v}\right)$ with $v=3 / 5$ and $S_{6}\left(S_{2}\right)=S_{2}^{6}$. Shown are absolute differences between exact $S_{4}^{\mathrm{ex}}\left(S_{2}\right)$ and $S_{6}^{\mathrm{ex}}\left(S_{2}\right)$ and these approximate relationships, $\triangle S_{n} \equiv$ $S_{n}^{\mathrm{ex}}-S_{n}\left(S_{2}\right)$ based on the uniaxial orientational distribution function $f(\boldsymbol{u}) \propto$ $\exp \left(\propto P_{2}(\boldsymbol{u} \cdot \boldsymbol{n})\right)$ of Maier-Saupe type [22](a special case of the so called 'Bingham distribution', cf. [28]). In that representation, the quadratic I and Doi closures, for example, have a $\left|\triangle S_{4}\right|$ as large as 0.15 at $S_{2}=0.6$. 
(16) yields the closure, parameterized by $v>0$,

$\boldsymbol{M}: \boldsymbol{a}_{[4]}=\frac{1-\left(1-S_{2}\right)^{v}}{S_{2}} \boldsymbol{M}: \overline{\boldsymbol{a}_{[2]} \boldsymbol{a}_{[2]}}$.

Using (4) we obtain the identities

$\boldsymbol{M}:\left\{\boldsymbol{a}_{(2)} \boldsymbol{a}_{(2)}\right\}_{\mathrm{sym}}=\frac{1}{3} \boldsymbol{M}: \boldsymbol{a}_{(2)} \boldsymbol{a}_{(2)}+\frac{2}{3} \boldsymbol{a}_{(2)} \cdot \boldsymbol{M} \cdot \boldsymbol{a}_{(2)}$,

$\boldsymbol{M}:\left\{\boldsymbol{a}_{(2)} \boldsymbol{I}\right\}_{\mathrm{sym}}$

$$
=\frac{1}{6}\left[\boldsymbol{M}: \boldsymbol{a}_{(2)} \boldsymbol{I}+2 \boldsymbol{a}_{(2)} \cdot \boldsymbol{M}+2 \boldsymbol{M} \cdot \boldsymbol{a}_{(2)}+\operatorname{tr}(\boldsymbol{M}) \boldsymbol{a}_{(2)}\right],
$$

$\boldsymbol{M}:\{\boldsymbol{I I}\}_{\mathrm{sym}}=\frac{1}{3}\left[\operatorname{tr}(\boldsymbol{M}) \boldsymbol{I}+\boldsymbol{M}+\boldsymbol{M}^{T}\right]$.

To facilitate comparison with the literature, and implementation in a computer code, we apply (23) to an anisotropic 2 nd rank tensor $\boldsymbol{A}=\boldsymbol{M}$, with $\operatorname{tr}(\boldsymbol{A})=0$ and $\boldsymbol{A}=\boldsymbol{A}^{T}$ and rewrite (23) with the help of (24) as

$$
\begin{aligned}
\boldsymbol{A}: \boldsymbol{a}_{(4)}= & \frac{1-\left(1-S_{2}\right)^{v}}{3 S_{2}}\left[\boldsymbol{A}: \boldsymbol{a}_{(2)} \boldsymbol{a}_{(2)}+2 \boldsymbol{a}_{(2)} \cdot \boldsymbol{A} \cdot \boldsymbol{a}_{(2)}\right]+\frac{\left(1-S_{2}\right)^{v}\left(1+(2 / 7) S_{2}\right)+S_{2}-1}{9 S_{2}}\left[\boldsymbol{A}: \boldsymbol{a}_{(2)} \boldsymbol{I}+4 \boldsymbol{a}_{(2)} \cdot \boldsymbol{A}\right] \\
& -\frac{\left((2 / 27)-(2 / 35) S_{2}^{2}\right)\left[\left(1-S_{2}\right)^{v}-1\right]+(2 / 27)\left[(1 / 5)+(4 / 7)\left(1-S_{2}\right)^{v}\right] S_{2}}{S_{2}} \boldsymbol{A},
\end{aligned}
$$

with $S_{2}$ also expressed in terms of $\boldsymbol{a}_{(2)}$ according to (13). We propose using (25) with constant $v>0$ in the uniaxial phase. It is correct in the isotropic and totally aligned limits and fulfills all constraints, nothing else can be concluded about the interrelation between $\boldsymbol{A}: \boldsymbol{a}_{(4)}$ and $\boldsymbol{A}$ and $\boldsymbol{a}_{(2)}$ without using a particular model or assumption. Notice, the three prefactors in (25) reduce to (i) $\frac{1}{3} \alpha=\frac{v}{3}-\frac{1}{6} v(v-1) S_{2}+o\left[S_{2}^{2}\right],-\frac{1}{3} \beta=\frac{1}{7}-\frac{v}{9}+o\left[S_{2}^{2}\right]$ and $-\frac{4}{3} \gamma=-\left(\frac{2}{35}-\frac{2}{27} \nu\right)+o\left[S_{2}^{2}\right]$, respectively, in the limit of small order parameter $S_{2}$, i.e., weak anisotropy and (ii) $\frac{1}{3} \alpha=\frac{1}{3}$, $-\frac{1}{3} \beta=\frac{2}{63}\left(1-S_{2}\right)$, and $-\frac{4}{3} \gamma=\frac{8}{945}\left(2+5 S_{2}-7 S_{2}^{2}\right)$ for the so called quadratic III closure $(v=1)$. See Table 1 for special choices of $\boldsymbol{M}$ or $\boldsymbol{A}$. It is trivial to check that (25) yields, for any $v$, the correct limiting cases: $\boldsymbol{A}: \boldsymbol{a}_{(4)}=\frac{2}{15} \boldsymbol{I}$ and $\boldsymbol{A}: \boldsymbol{a}_{(4)}=$ $\boldsymbol{A}: \boldsymbol{n}_{(4)}$ in the isotropic $\left(S_{2}=0\right)$, and totally aligned $\left(S_{2}=1\right)$, phase, respectively. For the special choice $\boldsymbol{M}=\boldsymbol{a}_{(2)}$, Eq. (5) can be used to replace $\boldsymbol{a}_{(2)}: \boldsymbol{a}_{(2)}$ by $\left(2 S_{2}^{2}+1\right) / 3$, and (25) can be also viewed as an expansion using the Caley-Hamilton theorem, where the coefficients are actually given in terms of the invariants of $\boldsymbol{a}_{(2)}$, cf. [9]. To this end notice the recursive rela-

Table 1

Some identities valid in the uniaxial phase, where $\boldsymbol{a}_{[2]}=S_{2} \boldsymbol{n}_{[2]}$

$\boldsymbol{a}_{[2]}:\left\{\boldsymbol{a}_{(2)} \boldsymbol{a}_{(2)}\right\}_{\mathrm{sym}}=\frac{2}{27}\left(1+2 S_{2}+6 S_{2}^{2}\right) \boldsymbol{a}_{[2]}+\frac{2}{81} S_{2}^{2}\left(7+2 S_{2}\right) \boldsymbol{I}$

$\boldsymbol{a}_{[2]}:\left\{\boldsymbol{a}_{(2)} \boldsymbol{I}\right\}_{\mathrm{sym}}=\frac{2}{9}\left(1+S_{2}\right) \boldsymbol{a}_{[2]}+\frac{7}{27} S_{2}^{2} \boldsymbol{I}$

$\boldsymbol{a}_{[2]}:\{\boldsymbol{I I}\}_{\mathrm{sym}}=\frac{2}{3} \boldsymbol{a}_{[2]}$

$\boldsymbol{a}_{(2)}:\left\{\boldsymbol{a}_{(2)} \boldsymbol{a}_{(2)}\right\}_{\mathrm{sym}}=\frac{1}{9}\left(3+2 S_{2}+4 S_{2}^{2}\right) \boldsymbol{a}_{[2]}+\frac{1}{81}\left(5+18 S_{2}^{2}+4 S_{2}^{3}\right) \boldsymbol{I}$

$\boldsymbol{a}_{(2)}:\left\{\boldsymbol{a}_{(2)} \boldsymbol{I} \boldsymbol{I}_{\mathrm{sym}}=\frac{1}{18}\left(11+4 S_{2}\right) \boldsymbol{a}_{[2]}+\frac{1}{27}\left(5+7 S_{2}^{2}\right) \boldsymbol{I}\right.$

$\boldsymbol{a}_{(2)}:\{\boldsymbol{I} \boldsymbol{I}\}_{\mathrm{sym}}=\frac{2}{3} \boldsymbol{a}_{[2]}+\frac{5}{9} \boldsymbol{I}$ tionship [9] between tensorial products $\boldsymbol{a}_{[2]}^{3}=\boldsymbol{a}_{[2]} \cdot \boldsymbol{a}_{[2]} \cdot \boldsymbol{a}_{[2]}$, etc.

$\boldsymbol{a}_{[2]}^{i}=I_{2} \boldsymbol{a}_{[2]}^{i-1}+I_{3} \boldsymbol{a}_{[2]}^{i-3}$

for $i \geq 3$ and with $\boldsymbol{a}_{[0]} \equiv \boldsymbol{I}$, where $I_{2}=\frac{1}{2} \boldsymbol{a}_{[2]}: \boldsymbol{a}_{[2]}$ and $I_{3}=$ $\operatorname{det}\left(\boldsymbol{a}_{[2]}\right)=\operatorname{tr}\left(\boldsymbol{a}_{[2]}^{3}\right)-I_{2} \boldsymbol{a}_{[2]}$ [9]. Using (26) we can, for example, write $\boldsymbol{a}_{(2)} \cdot \boldsymbol{a}_{(2)} \cdot \boldsymbol{a}_{(2)}$, which appears if $\boldsymbol{M}=\boldsymbol{a}_{(2)}$ is required, in terms of $\boldsymbol{I}, \boldsymbol{a}_{(2)}$, and $\boldsymbol{a}_{(2)} \cdot \boldsymbol{a}_{(2)}$, where the prefactors contain the invariants of $\boldsymbol{a}_{[2]}$.

\subsection{Comparison to closure relationships used in the literature}

(A) The so-called linear closure had been suggested by Hand [19], which is equivalent with (7) upon simply setting $\boldsymbol{a}_{[4]}=$ 0, i.e.,

$\boldsymbol{a}_{(4)}=\frac{6}{7}\left\{\boldsymbol{a}_{(2)} \boldsymbol{I}\right\}_{\mathrm{sym}}-\frac{3}{35}\{\boldsymbol{I} \boldsymbol{I}\}_{\mathrm{sym}}$

coincides with (16) for $S_{4}\left(S_{2}\right)=0$ which is however inconsistent with the required boundary condition $S_{4}(1)=1$. Hand's closure simply corresponds to $v=0$ in (21). For symmetric traceless $\boldsymbol{A}$ it predicts

$$
\boldsymbol{A}: \boldsymbol{a}_{(4)}=\frac{1}{7}\left[\boldsymbol{A}: \boldsymbol{a}_{(2)} \boldsymbol{I}+4 \boldsymbol{a}_{(2)} \cdot \boldsymbol{A}\right]-\frac{2}{35} \boldsymbol{A},
$$

which is a valid relationship in the isotropic phase, where it correctly reduces to $\boldsymbol{A}: \boldsymbol{a}_{(4)}=\frac{2}{15} \boldsymbol{A}$.

(B) The more general, so-called natural closure [32] reads:

$$
\begin{aligned}
\boldsymbol{a}_{(4)}= & \beta_{1}\{\boldsymbol{I} \boldsymbol{I}\}_{\mathrm{sym}}+\beta_{2}\left\{\boldsymbol{a}_{(2)} \boldsymbol{I}\right\}_{\mathrm{sym}}+\beta_{3}\left\{\boldsymbol{a}_{(2)} \boldsymbol{a}_{(2)}\right\}_{\mathrm{sym}} \\
& +\beta_{4}\left\{\boldsymbol{a}_{(2)} \cdot \boldsymbol{a}_{(2)} \boldsymbol{I}\right\}_{\mathrm{sym}}+\beta_{5}\left\{\boldsymbol{a}_{(2)} \boldsymbol{a}_{(2)} \cdot \boldsymbol{a}_{(2)}\right\}_{\mathrm{sym}} \\
& +\beta_{6}\left\{\boldsymbol{a}_{(2)} \cdot \boldsymbol{a}_{(2)} \boldsymbol{a}_{(2)} \cdot \boldsymbol{a}_{(2)}\right\}_{\mathrm{sym}}
\end{aligned}
$$

where the $\beta$ 's are functions of the second and third invariants of $\boldsymbol{a}_{(2)}$ and must be chosen to satisfy the normalization condition $\operatorname{tr}\left(\boldsymbol{a}_{(4)}\right)=\boldsymbol{a}_{(2)}$. By comparing (29) with (16) we see, that the functions $\beta_{1,2,3}$ are interrelated by our Eq. (17), and that functions $\beta_{4,5,6}$ vanish in the uniaxial phase. While the natural closure is somewhat general, our closure imposes constraints to its coefficients. The most general closure consistent with the Caley-Hamilton theorem and motivated by time-structure invariance criteria had been presented by Edwards and Öttinger [25]. It contains the natural closure as a special case. Their closure contains 27 scalar functions, cf. Section 5.1.

(C) The so-called quadratic I closure, $\boldsymbol{a}_{(4)}=\boldsymbol{a}_{(2)} \boldsymbol{a}_{(2)}$, is not compatible with the closure (16), except in the case of perfect 
alignment, $S_{4}=S_{2}=1$, and had been rarely used for the reason that it does produce very inconsistent results in the vicinity of the isotropic phase.

(D) The so called quadratic II closures, $\boldsymbol{a}_{[4]}=\boldsymbol{a}_{[2]} \boldsymbol{a}_{[2]}$ is inconsistent with our closure, though correct in the limit either for total alignment or random alignment.

(E) The 'quadratic III closure' which we introduce as $\boldsymbol{a}_{[4]}=$ $\boldsymbol{a}_{[2]} \boldsymbol{a}_{[2]}$, is a special parameter-free case of our closure for $v=1$. Replacing $S_{4}$ by $S_{2}^{2}$ in (17), (16) yields $\boldsymbol{a}_{[4]}=\widetilde{\boldsymbol{a}_{[2]} \boldsymbol{a}_{[2]}}$, or

$$
\begin{aligned}
\boldsymbol{a}_{(4)}= & \left\{\boldsymbol{a}_{(2)} \boldsymbol{a}_{(2)}\right\}_{\mathrm{sym}}-\frac{4}{21}\left(S_{2}-1\right)\left\{\boldsymbol{a}_{(2)} \boldsymbol{I}\right\}_{\mathrm{sym}} \\
& +\frac{4}{315}\left(2+5 S_{2}-7 S_{2}^{2}\right)\{\boldsymbol{I} \boldsymbol{I}\}_{\mathrm{sym}},
\end{aligned}
$$

which is, as a member of the set of allowed closures for $S_{2} \geq 0$, certainly valid both in the isotropic $\boldsymbol{a}_{(4)}=\frac{1}{5}\{\boldsymbol{I I}\}_{\text {sym }}$ and totally aligned phases $\boldsymbol{a}_{(4)}=\boldsymbol{n}_{(4)}$. The corresponding closure relationship for $\boldsymbol{A}: \boldsymbol{a}_{(4)}$ results from either (16) with $v=1$, or (30) with (24):

$$
\begin{aligned}
\boldsymbol{A}: \boldsymbol{a}_{(4)}= & \frac{1}{3}\left[2 \boldsymbol{a}_{(2)} \cdot \boldsymbol{A} \cdot \boldsymbol{a}_{(2)}+\boldsymbol{A}: \boldsymbol{a}_{(2)} \boldsymbol{a}_{(2)}\right. \\
& +\frac{8}{315}\left(2+5 S_{2}-7 S_{2}^{2}\right) \boldsymbol{A}-\frac{2}{21}\left(S_{2}-1\right)\left(\boldsymbol{A}: \boldsymbol{a}_{(2)} \boldsymbol{I}\right. \\
& \left.\left.+2 \boldsymbol{a}_{(2)} \cdot \boldsymbol{A}+2 \boldsymbol{A} \cdot \boldsymbol{a}_{(2)}\right)\right]
\end{aligned}
$$

It also seems reasonable to expect that relations (30) and (31) are good approximations in the case of weak biaxiality.

Two more commonly cited closures, motivated by Hinch and Leal [21] for an arbitrary symmetric and traceless tensor $\boldsymbol{A}$ are the HL-I and HL-II closures. These are based on interpolation between weak and strong flow limits in a Brownian suspension of rods.

(F) HL-I closure:

$$
\begin{aligned}
\boldsymbol{A}: \boldsymbol{a}_{(4)}= & \frac{1}{5}\left[6 \boldsymbol{a}_{(2)} \cdot \boldsymbol{A} \cdot \boldsymbol{a}_{(2)}-\boldsymbol{A}: \boldsymbol{a}_{(2)} \boldsymbol{a}_{(2)}\right. \\
& \left.+2 \boldsymbol{I}\left(\boldsymbol{a}_{(2)}-\boldsymbol{a}_{(2)} \cdot \boldsymbol{a}_{(2)}\right): \boldsymbol{A}\right],
\end{aligned}
$$

G HL-II closure:

$$
\begin{aligned}
\boldsymbol{A}: \boldsymbol{a}_{(4)}= & \boldsymbol{a}_{(2)} \boldsymbol{a}_{(2)}: \boldsymbol{A}+2 \boldsymbol{a}_{(2)} \cdot \boldsymbol{A} \cdot \boldsymbol{a}_{(2)} \\
& -\frac{2\left(\boldsymbol{a}_{(2)} \cdot \boldsymbol{a}_{(2)}\right): \boldsymbol{A}}{\boldsymbol{a}_{(2)}: \boldsymbol{a}_{(2)}} \boldsymbol{a}_{(2)} \cdot \boldsymbol{a}_{(2)} \\
& +\exp \left\{\frac{2-6 \boldsymbol{a}_{(2)}: \boldsymbol{a}_{(2)}}{1-\boldsymbol{a}_{(2)}: \boldsymbol{a}_{(2)}}\right\}\left[\frac{52}{315} \boldsymbol{A}-\frac{8}{21}\left(\boldsymbol{A} \cdot \boldsymbol{a}_{(2)}\right.\right. \\
& \left.\left.+\boldsymbol{a}_{(2)} \cdot \boldsymbol{A}-\frac{2}{3} \boldsymbol{a}_{(2)}: \boldsymbol{A I}\right)\right]
\end{aligned}
$$

Both the HL-I and HL-II closures are correct in the isotropic $\left(\boldsymbol{A}: \boldsymbol{a}_{(4)}=\frac{2}{15} \boldsymbol{A}\right)$ and totally aligned $\left(\boldsymbol{A}: \boldsymbol{a}_{(4)}=\right.$ $\boldsymbol{A n}(4))$ phases, and both are inconsistent with our closure (25) valid in the uniaxial phase.

(H) Hybrid closure: it has also been proposed [34,37] to use different closures for different terms in the same equation. For example, quadratic closure has been proposed for terms such as $\boldsymbol{a}_{(2)}: \boldsymbol{a}_{(4)}$ and the HL-I for $\boldsymbol{D}: \boldsymbol{a}_{(4)}$ where $\boldsymbol{D}$ is the symmetric part of the velocity gradient. This purely empirical approach is clearly inconsistent with our results.

(I) Doi [20] used the following closure relationship $\boldsymbol{a}_{[2]}: \boldsymbol{a}_{(4)}=$ $\boldsymbol{a}_{[2]}: \boldsymbol{a}_{(2)} \boldsymbol{a}_{(2)}$, which is equivalent with

$a_{(4)}: a_{(2)}=a_{(2)}: a_{(2)} a_{(2)}$

It is correct in the totally aligned phase, but not in the

\begin{tabular}{|c|c|c|c|c|}
\hline Uniaxial closure & Equation & $\boldsymbol{X} \equiv \boldsymbol{a}_{(2)} \cdot \boldsymbol{a}_{(2)}-\boldsymbol{a}_{(4)}: \boldsymbol{a}_{(2)}$ & iso & total \\
\hline Exact & (25) & $\frac{1}{35}\left(7+5 S_{2}-12 S_{4}\right) a_{[2]}$ & $\frac{1}{5}$ & 0 \\
\hline Exact, $S_{4}$ via (21) & $(25)$ & $\frac{1}{35}\left[7\left(1-S_{2}\right)+12 S_{2}\left(1-S_{2}\right)^{v}\right] \boldsymbol{a}_{[2]}$ & $\frac{1}{5}$ & 0 \\
\hline Linear (Hand) [19] & $(28)$ & $\frac{1}{35}\left(7+5 S_{2}\right) \boldsymbol{a}_{[2]}$ & $\frac{1}{5}$ & $\frac{12}{35}$ \\
\hline Doi [20], p. 359 & $(34)$ & $\frac{1}{3}\left(1+S_{2}-2 S_{2}^{2}\right) \boldsymbol{a}_{[2]}$ & $\frac{1}{3}$ & 0 \\
\hline Quadratic I: $\boldsymbol{a}_{(4)}=\boldsymbol{a}_{(2)} \boldsymbol{a}_{(2)}$ & & $\frac{1}{3}\left(1+S_{2}-2 S_{2}^{2}\right) \boldsymbol{a}_{[2]}$ & $\frac{1}{3}$ & 0 \\
\hline Quadratic II: $\boldsymbol{a}_{[4]}=\boldsymbol{a}_{[2]} \boldsymbol{a}_{[2]}$ & & $\left(\frac{19}{35}+\frac{1}{7} S_{2}-\frac{2}{3} S_{2}^{2}\right) \boldsymbol{a}_{[2]}+\frac{2}{7} \boldsymbol{I}$ & - & - \\
\hline Quadratic III: $\boldsymbol{a}_{[4]}=\boldsymbol{a}_{[2]} \boldsymbol{a}_{[2]}$ & (31) & $\frac{1}{35}\left(7+5 S_{2}-12 S_{2}^{2}\right) a_{[2]}$ & $\frac{1}{5}$ & 0 \\
\hline HL I closure (32) & $(32)$ & $\frac{1}{15}\left(3+S_{2}-4 S_{2}^{2}\right) \boldsymbol{a}_{[2]}$ & $\frac{1}{5}$ & 0 \\
\hline HL II closure (33) & (33) & {$\left[\frac{4\left(7+20 S_{2}\right)}{315} \mathrm{e}^{\left(6 S_{2}^{2} /\left(S_{2}^{2}-1\right)\right)}+\frac{\left(1-S_{2}+6 S_{2}^{2}+14 S_{2}^{3}-20 S_{2}^{4}\right)}{9\left(1+2 S_{2}^{2}\right)}\right] \boldsymbol{a}_{[2]}$} & $\frac{1}{5}$ & 0 \\
\hline
\end{tabular}
isotropic phase and thus incompatible with (25).

In the special case of isotropy and perfect uniaxial alignment any closure with correct tensorial symmetry must reduce to $\frac{2}{15} \boldsymbol{A}$, and $\boldsymbol{A}: \boldsymbol{n}_{(4)}$, respectively (for any anisotropic $\boldsymbol{A}$ ). Further, $\operatorname{tr}(\boldsymbol{A}$ : $\left.\boldsymbol{a}_{(4)}\right)=\boldsymbol{A}: \boldsymbol{a}_{(2)}$ must hold. Together with the test of limiting cases, the latter constraint serves as an independent test of a possible useful closure relationship, cf. Table 2 for details and Fig. 2 for a graphical representation.

Table 2

Comparison of closure relationships for the uniaxial phase (director $\boldsymbol{n}$ )

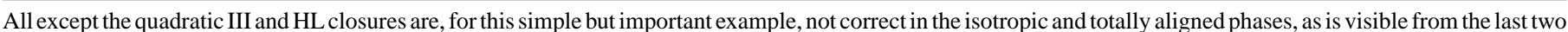

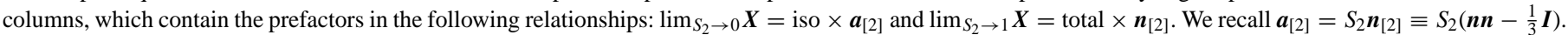
See Fig. 2 for a graphical representation. 


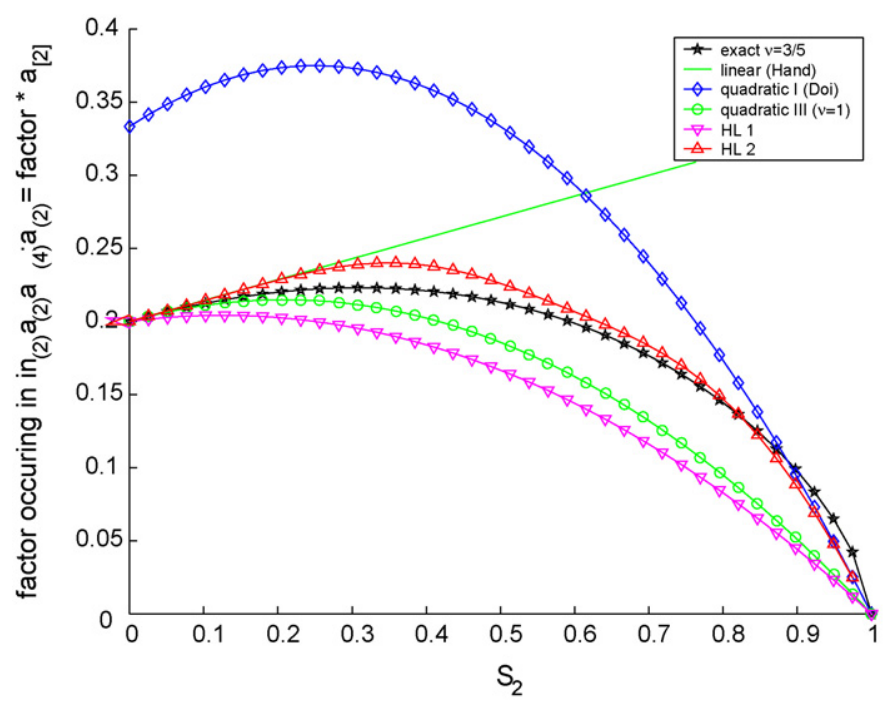

Fig. 2. Comparison of decoupling approximations, cf. Tables 2 and 3.

\subsection{Potential applications}

\subsubsection{Liquid crystals}

Let us consider the Hess-Doi theory $[10,20]$ for rigid, rodlike liquid crystal polymers. Consider a flow with macroscopic velocity $\mathbf{v}$. The symmetric $\boldsymbol{D}$ and antisymmetric parts $\boldsymbol{W}$ of the velocity gradient are given by $\boldsymbol{D}=\frac{1}{2}\left[\nabla \mathbf{v}+(\nabla \mathbf{v})^{T}\right], \boldsymbol{W}=$ $-\frac{1}{2}\left[\nabla \mathbf{v}-(\nabla \mathbf{v})^{T}\right]$. The interaction of the rods is modeled by a mean field contribution $\nabla \boldsymbol{u} V_{\mathrm{mf}}$ where $V_{\mathrm{mf}}=-\frac{3}{2} U k_{\mathrm{B}} T \boldsymbol{u}_{(2)}$ : $\boldsymbol{a}_{(2)}$, with $U$ the strength of the mean field. Here the mean field imposes uniaxial symmetry in equilibrium. The flow can however induce biaxiality, but for small shear rates this biaxiality is weak. The evolution equation for the second moment $\boldsymbol{a}_{(2)}$ is

$$
\begin{aligned}
\frac{\partial \boldsymbol{a}_{(2)}}{\partial t}+\mathbf{v} \cdot \nabla \boldsymbol{a}_{(2)}= & \boldsymbol{W} \cdot \boldsymbol{a}_{(2)}-\boldsymbol{a}_{(2)} \cdot \boldsymbol{W}+R\left(\boldsymbol{D} \cdot \boldsymbol{a}_{(2)}+\boldsymbol{a}_{(2)} \cdot \boldsymbol{D}\right) \\
& -2 R \boldsymbol{D}: \boldsymbol{a}_{(4)}-6 D_{\mathrm{r}} \boldsymbol{a}_{[2]}+6 U D_{\mathrm{r}}\left(\boldsymbol{a}_{(2)}\right. \\
& \left.\cdot \boldsymbol{a}_{(2)}-\boldsymbol{a}_{(4)}: \boldsymbol{a}_{(2)}\right)
\end{aligned}
$$

where $D_{\mathrm{r}}$ is the rotary diffusion coefficient and $R$ a shape coefficient characterizing rods $(\lambda=1)$, spheres $(\lambda=0)$ and disks $(\lambda=-1)$. We can substitute expression (25) for $\boldsymbol{D}: \boldsymbol{a}_{(2)}$ and $\boldsymbol{a}_{(4)}: \boldsymbol{a}_{(2)}$ to obtain a closed evolution equation for $\boldsymbol{a}_{(2)}$, parameterized by $v$. For $v=1$ one can use (31) instead of (25). The same equations are commonly used for dilute suspensions of rigid particles, polymers, etc. but where now $U=0[14,36,38,39]$. For explicit expressions of $\boldsymbol{a}_{(2)} \cdot \boldsymbol{a}_{(2)}-\boldsymbol{a}_{(4)}: \boldsymbol{a}_{(2)}$ in terms of order parameters see Table 2, $\boldsymbol{D}: \boldsymbol{a}_{(4)}$ is given by (25) upon replacing $\boldsymbol{A}$ by $\boldsymbol{D}$ because $\boldsymbol{D}$ is symmetric and traceless (incompressible flow). For the case of compressible flows, one has to use the more general equations containing $\operatorname{tr}(\boldsymbol{M})$ of this manuscript.

Doi [20] considered the flow-free relaxation behavior $(\boldsymbol{D}=$ $\boldsymbol{W}=\mathbf{0})$ of a liquid crystal, assumed to be uniaxially preoriented (in direction $\boldsymbol{n}$ ) by an external field (at time $t=0$ ). Hence, we can insert the ansatz $\boldsymbol{a}_{[2]}(t)=S_{2}(t) \boldsymbol{n}_{[2]}$ into (35) to obtain an equation of change for the scalar order parameter $S_{2}(t): \frac{\mathrm{d} S_{2}}{\mathrm{~d} t}=-6 D_{\mathrm{r}} S_{2}+6 U D_{\mathrm{r}} X$, where $X$ defined as $\boldsymbol{X}=$
$X \boldsymbol{n}_{[2]}$ depends on the closure relationship and is tabulated in Table 2 for various models. Accordingly, the closure (16) yields $\mathrm{d} S_{2} / \mathrm{d} t=-6 D_{\mathrm{r}} \partial A\left(S_{2}, U\right) / \partial S_{2}$ with an associated free energy

$$
\begin{aligned}
A\left(S_{2}, U\right)= & \frac{1}{2}\left(1-\frac{U}{5}\right) S_{2}^{2}+\frac{U}{15} S_{2}^{3} \\
& +\frac{12\left(1-S_{2}\right)^{1+v}\left\{2+(1+v)\left(2+[2+v] S_{2}\right) S_{2}\right\} U}{35(1+v)(2+v)(3+v)}
\end{aligned}
$$

$$
\begin{aligned}
= & \frac{1}{2}\left(1-\frac{U}{5}\right) S_{2}^{2}-\frac{U}{21} S_{2}^{3}+\frac{3 v U}{35} S_{2}^{4}-\frac{6 v(v-1) U}{175} S_{2}^{5} \\
& +v(v-1) o\left[S_{2}^{6}\right],
\end{aligned}
$$

to be compared with $A\left(S_{2}, U\right)=\frac{1}{2}(1-U / 3) S_{2}^{2}-(U / 9) S_{2}^{3}+$ $(U / 6) S_{2}^{4}$ of [20]. For an amended potential (37) that not only requires, but ensures the constraint $S_{2} \leq 1$ in strong flows, for example, we refer the reader to [40]. Notice that the term proportional to $S_{2}^{5}$ in (37) vanishes only for $v=1$. For this choice, and $U<U_{\mathrm{NI}} \equiv 1680 / 361 \approx 4.653$ ([20] predicts $U_{\mathrm{NI}}=2.667$ instead, $U_{\mathrm{NI}}=4.898,4.457$ for the HL-I and HL-II closure, respectively, and $U_{\mathrm{NI}}=4.55$ for the unapproximated theory), $A$ has only one minimum at $S_{2}=0$, so that the system finally becomes isotropic, whereever its initial state. For $U_{\mathrm{NI}}<U<$ $U_{c} \equiv 5$ ([20] predicts $U_{c}=3$ ), there are two local minima, one at $S_{2}=0$ and the other at

$S_{\mathrm{eq}}=\frac{5}{24}+\frac{19}{24}\left[1-\left(U_{\mathrm{NI}} / U\right)\right]^{1 / 2}$,

to be compared with $S_{\text {eq }}=\frac{1}{4}+\frac{3}{4}[1-8 /(3 U)]^{1 / 2}$ of [20]. The above relationships can be also worked out for arbitrary $\nu$, or in terms of $S_{4}$. For completeness, we mention the equilibrium order parameter which results from the approximate expression (37): $S_{\mathrm{eq}}=\left[2^{1 / 3} H^{2}-4 H \nu U+2^{2 / 3} v(5+3 v) U^{2}\right] /[6 H v(1-$ v) $U$ ] with $H \equiv U^{2 / 3}\left(v^{3 / 2} \sqrt{G}+v^{2} F\right)^{1 / 3}$, and $G \equiv v F^{2}-$ $2(5+3 v)^{3} U^{2}$, and $F \equiv[33-v(128-63 v)] U-315(1-v)^{2}$. This formula exactly reduces to (38) for $v=1$ and $U \geq U_{\mathrm{NI}}$ and allows to estimate $v$ from a measured $S_{\text {eq }}$. Generally, $S_{\text {eq }}$ tends to increase with decreasing $v$. For $v=0$, i.e. $S_{4}=0, U_{\mathrm{NI}}=5$ and $U_{c}$ does not exist, cf. Fig. 3 and Table 3, which contains

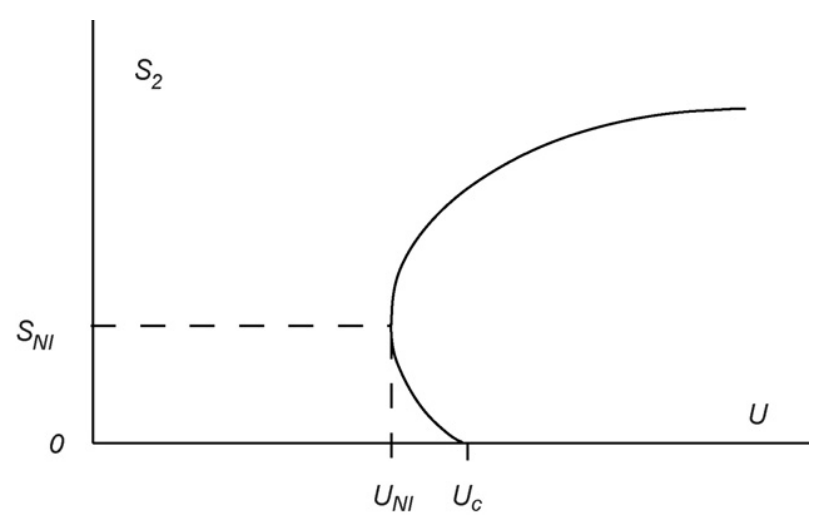

Fig. 3. Definition of $U_{\mathrm{NI}}, S_{\mathrm{NI}}$ and $U_{c}$, listed in Table 3. 
Table 3

Comparison of closure relationships

\begin{tabular}{lllll}
\hline Closure scheme & Equation & $U_{\mathrm{NI}}$ & $U_{c}$ & $S_{\mathrm{NI}}$ \\
\hline Quadratic I and Doi & $(34)$ & $\frac{8}{3} \approx 2.67$ & 3 & $\frac{1}{4}=0.25$ \\
Linear (Hand) & $(28)$ & $\frac{35}{12} \approx 2.92$ & 5 & 1.00 \\
Consistent $(v=3 / 5)$ & $(25)$ & $\approx 4.48$ & 5 & $\approx 0.31$ \\
Consistent $(v=7 / 10)$ & $(25)$ & $\approx 4.54$ & 5 & $\approx 0.28$ \\
Quadratic III $(v=1)$ & $(25)$ & $\frac{1680}{361} \approx 4.65$ & 5 & $\frac{5}{24} \approx 0.21$ \\
HL I & $(32)$ & $\frac{240}{49} \approx 4.90$ & 5 & $\frac{1}{8} \approx 0.13$ \\
HL II & $(33)$ & $\approx 4.38$ & 5 & $\approx 0.30$ \\
\hline
\end{tabular}

Characteristic parameters $U_{\mathrm{NI}}, U_{c}$, and $S_{\mathrm{NI}}$ introduced in Fig. 3. $U_{\mathrm{NI}}$, and $S_{\mathrm{NI}}$ are the values at the nematic-isotropic phase transition, and $U_{c}$, is the value above which the isotropic phase is no longer stable. The numerical solution $[27,46,48]$ gives $U_{\mathrm{NI}} \approx 4.49, U_{c}=5$, and $S_{\mathrm{NI}} \approx 0.3$ in nice agreement with the recommended consistent closure with $v=3 / 5$ (called 'consistent' in table); $U_{\mathrm{NI}}=4.898,4.457$ for the HL-I and HL-II closure, respectively, and $U_{\mathrm{NI}}=4.55$ for the unapproximated theory [12].

reference results for various closure schemes, and values from "exact" (numerical) solution.

\subsubsection{Ferrofluids and magnetorheological fluids}

As discussed in [5-7,20,41,42], the equation of change for the first moment $\boldsymbol{a}_{(1)}=\langle\boldsymbol{u}\rangle=S_{1} \boldsymbol{n}$ of the orientational distribution function of dipolar units in a ferrofluid subjected to a (dimensionless) magnetic field $\boldsymbol{h}$ reads

$$
\begin{aligned}
\frac{\mathrm{d}}{\mathrm{d} t} \boldsymbol{a}_{(1)}= & \boldsymbol{W} \cdot \boldsymbol{a}_{(1)}+R \boldsymbol{D} \cdot \boldsymbol{a}_{(1)}-R \boldsymbol{D}: \boldsymbol{a}_{(3)}-2 D_{\mathrm{r}} \boldsymbol{a}_{(1)} \\
& +D_{\mathrm{r}}\left(\boldsymbol{I}-\boldsymbol{a}_{(2)}\right) \cdot \boldsymbol{h}
\end{aligned}
$$

The closure relationships corresponding to (16) and (25) for the second and third moments read

$$
\begin{aligned}
& \boldsymbol{a}_{(2)}=\frac{S_{2}}{S_{1}^{2}} \boldsymbol{a}_{(1)} \boldsymbol{a}_{(1)}+\frac{1}{3}\left(1-S_{2}\right) \boldsymbol{I}, \\
& \boldsymbol{A}: \boldsymbol{a}_{(2)}=\frac{S_{2}}{S_{1}^{2}} \boldsymbol{A}: \boldsymbol{a}_{(1)} \boldsymbol{a}_{(1)}, \\
& \boldsymbol{a}_{(3)}=\frac{S_{3}}{S_{1}^{3}} \boldsymbol{a}_{(1)} \cdot \boldsymbol{a}_{(1)} \cdot \boldsymbol{a}_{(1)}+\frac{3}{5}\left\{\boldsymbol{I} \boldsymbol{a}_{(1)}\right\}_{\mathrm{sym}}\left(1-\frac{S_{3}}{S_{1}}\right), \\
& \boldsymbol{A}: \boldsymbol{a}_{(3)}=\frac{S_{3}}{S_{1}^{3}} \boldsymbol{A}: \boldsymbol{a}_{(1)} \boldsymbol{a}_{(1)} \boldsymbol{a}_{(1)}+\frac{2}{5} \boldsymbol{A} \cdot \boldsymbol{a}_{(1)}\left(1-\frac{S_{3}}{S_{1}}\right) .
\end{aligned}
$$

In the absence of flow, $S_{2}$ and $S_{3}$ can be expressed in terms of either $h$ or $S_{1}$ via (22) and this relationship should be kept in a first approximation when solving the equations in the presence of both flow and magnetic fields. It is known from simulation studies that biaxiality is weak in wide range of field strengths. In the absence of flow, of course, the ferrofluid is strictly in the uniaxial phase with $\boldsymbol{h}=h \boldsymbol{n}$, and (39) reduces to

$\frac{\mathrm{d}}{\mathrm{d} t} S_{1}=-2 D_{\mathrm{r}}\left[S_{1}-\frac{h}{3}\left(1-S_{2}\right)\right]$,

which is in agreement with (22), i.e., reduces to $\mathrm{d} S_{1} / \mathrm{d} t=0$ in equilibrium as it should. At the same time, we have hereby demonstrated how to actually derive the recursive relationship (22) in an alternate fashion, via closure relationships with correct tensorial symmetry.

\subsubsection{Landau-de Gennes potential}

The often quoted Landau-de Gennes potential $\Phi$, a scalar function in terms of the two nonvanishing invariants of $\boldsymbol{a}_{[2]}$, reads $[9,10,15,16,43,44]$

$\Phi=\Phi\left(S_{2}, I^{(3)}\right)=\frac{A}{3} \zeta_{2} S_{2}^{2}-\frac{B}{3} \zeta_{2}^{2} I^{(3)}+\frac{C}{9} \zeta_{2}^{3} S_{2}^{3}$,

with $I^{(3)} \equiv \sqrt{6} \operatorname{tr} \boldsymbol{a}_{[2]}^{3}=3 \sqrt{6} \operatorname{det} \boldsymbol{a}_{[2]} \quad$ [9] and orientationindependent coefficients $A, B$, and $C$, such that

$$
\begin{aligned}
\boldsymbol{\Phi}\left(\boldsymbol{a}_{[2]}\right) & =\frac{\partial \Phi}{\partial \boldsymbol{a}_{[2]}} \\
& =A \zeta_{2} \boldsymbol{a}_{[2]}-\sqrt{6} B \zeta_{2}^{2} \overline{\boldsymbol{a}_{[2]} \cdot \boldsymbol{a}_{[2]}}+C \zeta_{2}^{3} \boldsymbol{a}_{[2]} \boldsymbol{a}_{[2]}: \boldsymbol{a}_{[2]},
\end{aligned}
$$

where $\zeta_{2}=\sqrt{15 / 2}$ according to (2), and the related equation of change for $\boldsymbol{a}_{[2]}$ is usually written in the form [16]

$$
\begin{aligned}
\frac{\partial}{\partial t} \boldsymbol{a}_{[2]}= & 2 \longdiv { \boldsymbol { \omega } \times \boldsymbol { a } _ { [ 2 ] } } + 2 \sigma \widetilde { \boldsymbol { D } \cdot \boldsymbol { a } _ { [ 2 ] } } - \tau _ { a } ^ { - 1 } \zeta _ { 2 } ^ { - 1 } \boldsymbol { \Phi } ( \boldsymbol { a } _ { [ 2 ] } ) \\
& -\sqrt{2} \zeta_{2}^{-1} \frac{\tau_{\mathrm{ap}}}{\tau_{\mathrm{a}}} \boldsymbol{D},
\end{aligned}
$$

with vorticity $\boldsymbol{\omega}=\frac{1}{2} \nabla \times \boldsymbol{v}$. For incompressible flow, $\boldsymbol{D}=\boldsymbol{D}$. By comparing (47) with (35) we see that $\sigma=R$. Matching the isotropic phase where $\boldsymbol{a}_{(4)}=\frac{1}{5}\{\boldsymbol{I I}\}_{\text {sym }}$, we obtain $\tau_{\mathrm{ap}} / \tau_{\mathrm{a}}=$ $2 R / \sqrt{15}$. The two equations, however, do not match in the case of perfect alignment $\left(\boldsymbol{a}_{[2]}=\boldsymbol{n}_{[2]}\right)$, since the last term in (47) has no analogue in (35), and because the coefficients $A, B$, and $C$ in (47) are assumed to be orientation-independent. For weak flows, however, we can equate (35) with (47), to obtain

$$
\begin{aligned}
U \boldsymbol{X}= & \boldsymbol{a}_{[2]}-\frac{1}{6 D_{\mathrm{r}} \tau_{\mathrm{a}}} \\
& \times\left(A \boldsymbol{a}_{[2]}-\sqrt{6} B \zeta_{2} \overline{\boldsymbol{a}_{[2]} \cdot \boldsymbol{a}_{[2]}}+C \zeta_{2}^{2} \boldsymbol{a}_{[2]} \boldsymbol{a}_{[2]}: \boldsymbol{a}_{[2]}\right),
\end{aligned}
$$

where we know from Table 2 that $\boldsymbol{X} \rightarrow \frac{1}{5} \boldsymbol{a}_{[2]}$ in the vicinity of the isotropic phase. In the uniaxial phase, where $I^{(3)}=\left(\frac{2 S_{2}}{3}\right)^{3}$ holds, and $\Phi=\Phi\left(S_{2}\right)$, Eq. (48) reduces, without approximations, to the scalar equation

$$
\begin{aligned}
& U \frac{1}{35}\left(7+5 S_{2}-12 S_{4}\right) \\
& \quad=1-\frac{1}{6 D_{\mathrm{r}} \tau_{\mathrm{a}}}\left(A-\sqrt{6} B \zeta_{2} S_{2} \frac{1}{3}+C \zeta_{2}^{2} \frac{2}{3} S_{2}^{2}\right),
\end{aligned}
$$

which allows, depending on the closure relationship, i.e. for any $v$, to identify the parameters of the Landau-de Gennes potential in terms of the Hess-Doi parameters. For the quadratic 
III closure $(v=1)$, for example, we have, with the constants $\chi \equiv \frac{6}{5} D_{\mathrm{r}} \tau_{\mathrm{a}}$, and $U_{\mathrm{c}}=5$ (cf. Table 3$)$,

$A=\chi\left(U_{\mathrm{c}}-U\right), \quad B=\frac{\sqrt{5}}{7} \chi, \quad C=\frac{12}{35} \chi$.

We have thus illustrated how to determine the free parameters of the Landau-de Gennes potential from a 'microscopic' FP approach. An alternate approach has been discussed in [16].

\section{Biaxial phase}

Two scalar order parameters $S_{2}$ and $B_{2}$ are required to uniquely characterize the second rank tensor $\boldsymbol{a}_{(2)}=\langle\boldsymbol{u} \boldsymbol{u}\rangle$ in the (most general) biaxial phase; these can be extracted from the sorted eigenvalues $\lambda_{1} \geq \lambda_{2} \geq \lambda_{3}$ and corresponding orthonormal eigenvectors $\boldsymbol{e}_{1}, \boldsymbol{e}_{2}$, and $\boldsymbol{e}_{3}=\boldsymbol{e}_{1} \times \boldsymbol{e}_{2}$ of $\boldsymbol{a}_{(2)}=\sum_{i} \lambda_{i} \boldsymbol{e}_{i} \boldsymbol{e}_{i}$ as follows:

$S_{2} \equiv \frac{1}{2}\left(3 \lambda_{1}-1\right), \quad \boldsymbol{n} \equiv \boldsymbol{e}_{1}$,

$B_{2} \equiv \lambda_{2}-\lambda_{3}, \quad \boldsymbol{m} \equiv \boldsymbol{e}_{2}$

where $\boldsymbol{n}$ and $\boldsymbol{m}$ are the 'directors', $B_{2}$ characterizes departures from the uniaxial phase. The order parameters $S_{2}$ and $B_{2}$ (both semipositive), the directors $\boldsymbol{n}$ and $\boldsymbol{m}$ are uniquely obtained from a given 2nd rank alignment tensor. Further $\lambda_{3}=$ $1-\lambda_{1}-\lambda_{2}$ holds since $\operatorname{tr}\left(\boldsymbol{a}_{(2)}\right)=1$. The (symmetric traceless) 2nd rank alignment tensor $\boldsymbol{a}_{[2]}=\boldsymbol{a}_{(2)}-\frac{1}{3} \boldsymbol{I}$ appearing in (8) can be written in terms of order parameters and directors $\boldsymbol{n}$ and $\boldsymbol{m}$ via anisotropic dyadic products $\boldsymbol{n}_{[2]} \equiv \boldsymbol{n n}-\frac{1}{3} \boldsymbol{I}$ and $\boldsymbol{m}_{[2]} \equiv \boldsymbol{m} \boldsymbol{m}-\frac{1}{3} \boldsymbol{I}$ as

$\boldsymbol{a}_{[2]}=\left(S_{2}+\frac{B_{2}}{2}\right) \boldsymbol{n}_{[2]}+B_{2} \boldsymbol{m}_{[2]}$.

Similarly, the (symmetric traceless) 4th rank alignment tensor $\boldsymbol{a}_{[4]}=\langle\overrightarrow{\boldsymbol{u} \boldsymbol{u} \boldsymbol{u} \boldsymbol{u}}\rangle$ reads, with anisotropic tensorial products $\boldsymbol{n}_{[4]}$, $\boldsymbol{m}_{[4]}$, and $\overline{\mathbf{n n m m}}$

$\boldsymbol{a}_{[4]}=\left(S_{4}-\frac{3 B_{4}}{8}+\frac{M_{4}}{2}\right) \boldsymbol{n}_{[4]}+B_{4} \boldsymbol{m}_{[4]}+M_{4} \overline{\boldsymbol{n n m m}}$,

with three order parameters $S_{4}, B_{4}, M_{4}$, and the directors $\boldsymbol{n}$ and $\boldsymbol{m}$ available from the second moment, characterizing the fourth moment. Explicit expressions for the quantities appearing in (53) are given in Appendix A, and not needed to simply use any of the closure relationships to be derived and presented in this section. It is however, important to notice, that $\boldsymbol{a}_{[4]}$ is written in terms of the eigenvectors characterizing the second moment. As for the order parameters of the 2nd rank tensor $\boldsymbol{a}_{[2]}$, the order parameters for the fourth rank tensor can be calculated for given $\boldsymbol{a}_{[4]}$ directly from (53) by suitable projection, or more conveniently, from Legendre polynomials.

A closure relationship (which expresses $\boldsymbol{a}_{[4]}$ in terms of $\boldsymbol{a}_{[2]}$ ) with correct tensorial symmetry must therefore express $S_{4}, B_{4}$, $M_{4}$ in terms of $S_{2}$ and $B_{2}$. Moreover, these functional dependencies must reduce to (i) $S_{4}=B_{4}=M_{4}=0$ for $S_{2}=B_{2}=0$ (isotropic phase), to (ii) $S_{4}=1, B_{2}=B_{4}=M_{4}=0$ for $S_{2}=1$ (totally aligned phase), and the order parameters are subject to inequalities and bounds as discussed in Appendix A. Insofar, (53) constitutes the most general closure relationship, but is not very useful in its present form because the irreducible tensors have still to be calculated, and usually contracted, cf. (35), with a symmetric traceless matrix $\boldsymbol{D}$. By replacing $\boldsymbol{D}$ in the resulting expression by $\boldsymbol{a}_{(2)}$ we have immediate access to $\boldsymbol{X} \equiv \boldsymbol{a}_{(2)} \cdot \boldsymbol{a}_{(2)}-\boldsymbol{a}_{(4)}: \boldsymbol{a}_{(2)}$, also appearing in (35). Generally valid identities for these quantities needed to implement a consistent biaxial closure are summarized in Appendix B. The eigenvector $\boldsymbol{e}_{3}$ does not appear in (52) and (53) because we eliminated it using the identity $\boldsymbol{I}=\sum_{i=1}^{3} \boldsymbol{e}_{i} \boldsymbol{e}_{i}$.

\subsection{Simple closures for biaxial symmetry}

In the biaxial case there are three independent scalar order parameters that must be determined. Thus, we obtain a consistent closure by postulating relations for $S_{4}, B_{4}$, and $M_{4}$ in terms of the second-order ones $S_{2}$ and $B_{2}$ :

$S_{4}=S_{4}\left(S_{2}, B_{2}\right), \quad B_{4}=B_{4}\left(S_{2}, B_{2}\right), \quad M_{4}=M_{4}\left(S_{2}, B_{2}\right)$.

Note, however, that in general such relations do not provide an expression for the fourth-order alignment tensor in terms of the second-order alignment tensor. Only special choices of (54) will yield such expressions.

The three functions $S_{4}, B_{4}$, and $M_{4}$ are subject to several boundary conditions summarized after Eq. (A.7) which gives rise to these conditions. Rather than just fitting the biaxial order parameters to polynomials in $S_{2}$ and $B_{2}$ which do not necessarily respect the boundary conditions, one can start from a distribution function to derive consistent relationships, much in the same spirit as demonstrated for the uniaxial case where we introduced a parameterized relationship between $S_{4}$ and $S_{2}$. As we have seen above, closure relationships are in use which do not obey tensorial symmetries. Such inconsistent relationships can be recognized to eventually work fine in certain flow geometries, while failing in others. Closures expressed in the form (54) do not possess these problem.

\subsection{Quadratic-III closure for biaxial symmetry}

The only consistent, and parameter-free quadratic closure reads $\boldsymbol{a}_{[4]}=\widehat{\boldsymbol{a}_{[2]} \boldsymbol{a}_{[2]}}$ (quadratic-III) which corresponds to

$S_{4}=S_{2}^{2}+\frac{1}{8} B_{2}^{2}, \quad B_{4}=B_{2}^{2}, \quad M_{4}=2 S_{2} B_{2}+B_{2}^{2}$,

and certainly reduces to $S_{4}=S_{2}^{2}$ in the uniaxial phase. Substitution of (55) into (53) leads to an expression for $\boldsymbol{a}_{[4]}$ in terms of directors and order parameters satisfying $\boldsymbol{a}_{[4]}=\boldsymbol{a}_{[2]} \boldsymbol{a}_{[2]}$.

These special relations (55) are always consistent and yield the correct limits for perfect alignment (uniaxial) and random alignment (isotropic).

\section{3. $K$-I closure}

As the relationship $S_{4}=S_{2}^{2}$ is usually not valid even in equilibrium, we propose the following K-I closure for the biaxial 
phase

$\boldsymbol{a}_{[4]}=\zeta \boldsymbol{a}_{[2]} \boldsymbol{a}_{[2]}$,

with a scalar prefactor $\zeta$ which is introduced to represent the ratio between $S_{4}$ and $S_{2}^{2}$ obtained from the (usually known) equilibrium distribution function. This closure relationship (56) with any scalar $\zeta=\zeta\left(S_{2}\right)$ obeying $S_{2}^{2} \zeta=S_{2}$ for $S_{2}=0$ (isotropic) and $S_{2}=1$ (totally aligned), as well as $S_{2}^{2} \zeta<1$, has correct tensorial symmetry and is again a special case of the general closure (54). More precisely, closure K-I (56) is equally represented by

$S_{4}=\left(S_{2}^{2}+\frac{1}{8} B_{2}^{2}\right) \zeta$,

$B_{4}=B_{2}^{2} \zeta$

$M_{4}=\left(2 S_{2} B_{2}+B_{2}^{2}\right) \zeta$.

This K-I closure can be applied (and will be applied in Section 6 to flowing nematics) by using the basic identities summarized in Appendix B. Further identities, if needed, can be derived using the expressions of Appendix A, or by evaluating the irreducible tensor $\overline{\boldsymbol{a}_{[2]} \boldsymbol{a}_{[2]}}$ explicitly [9].

As discussed already for the uniaxial phase, for the case of a Maier-Saupe potential, we have access to the equilibrium relationship between $S_{4}$ and $S_{2}$ which allows to calculate $\zeta=\zeta\left(S_{2}\right)$ self-consistently from the orientational distribution function $f(\boldsymbol{u})$ of the (Maier-Saupe) form $f(\mathbf{u})=Z^{-1} \mathrm{e}^{\alpha P_{2}(\boldsymbol{u} \cdot \boldsymbol{n})}$ with normalization constant $Z$, yielding

$\zeta \equiv \frac{1-\left(1-S_{2}\right)^{v}}{S_{2}}, \quad v \approx \frac{3}{5}$.
The weighting factor $\zeta$ of (58) possesses the properties $\zeta=v$ for $v=0$ and $v=1$, more generally,

$\zeta=1+(v-1)\left[1-\frac{v}{2} S_{2}+\frac{v}{6}(v-2) S_{2}^{2}\right]+O\left[S_{2}^{3}\right]$.

That is, a maximum entropy argument has been used to relate $\left\langle P_{2}(\boldsymbol{u} \cdot \boldsymbol{n})\right\rangle \equiv \int f(\boldsymbol{u}) P_{2}(\boldsymbol{u} \cdot \boldsymbol{n}) d^{2} u$ and $\left\langle P_{4}(\boldsymbol{u} \cdot \boldsymbol{n})\right\rangle-$ with Legendre polynomials $P_{2}$ and $P_{4}-$ not only qualitatively, but accurately near equilibrium, more precisely, in the uniaxial phase. The biaxial K-I closure is therefore accurately valid both close to equilibrium, as well as in the aligned state, and offers correct tensorial symmetry, by construction.

\subsection{K-II closure}

Another simple closure, K-II, fullfilling all the criteria of closure K-I, is

$S_{4}=\left(S_{2}^{2}+\frac{1}{8} B_{2}^{2}\right) \zeta$

$B_{4}=B_{2}^{2}$,

$M_{4}=2 S_{2} B_{2}+B_{2}^{2}$,

with $\zeta=S_{4} / S_{2}^{2}$ to be (again) either approximated via (58) or to be - more generally - obtained from the equilibrium distribution function.

One can test the decoupling approximation by (i) solving a closed dynamical equation for the alignment tensor, or by (ii) verifying the relationships between order parameters such as (60), if a numerical solution to the underlying FP equation is available. To facilitate approach (ii), the order parameters are

Table 4

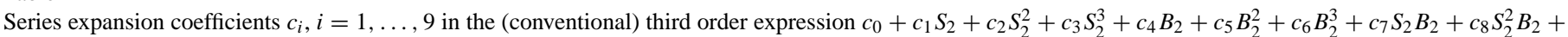

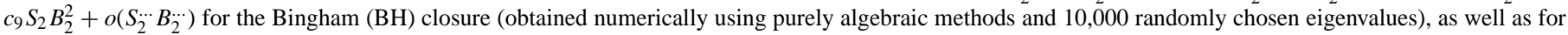
the K-I (arbitrary $v)$, K-I $(v=3 / 5)$ and K-II $(v=3 / 5)$ closures [obtained analytically from Eqs. (57) and (60)]

\begin{tabular}{|c|c|c|c|c|c|c|c|c|c|c|c|}
\hline Closure & $\begin{array}{l}c_{0} \\
1\end{array}$ & $\begin{array}{l}c_{1} \\
S_{2}\end{array}$ & $\begin{array}{l}c_{2} \\
S_{2}^{2}\end{array}$ & $\begin{array}{l}c_{3} \\
S_{2}^{3}\end{array}$ & $\begin{array}{l}c_{4} \\
B_{2}\end{array}$ & $\begin{array}{l}c_{5} \\
B_{2}^{2}\end{array}$ & $\begin{array}{l}c_{6} \\
B_{2}^{3}\end{array}$ & $\begin{array}{l}c_{7} \\
S_{2} B_{2}\end{array}$ & $\begin{array}{l}c_{8} \\
S_{2}^{2} B_{2}\end{array}$ & $\begin{array}{l}c_{9} \\
S_{2} B_{2}^{2}\end{array}$ & $\begin{array}{l}c_{\ldots} \\
S_{2} B_{2}\end{array}$ \\
\hline \multicolumn{12}{|l|}{$S_{4}$} \\
\hline $\mathrm{BH}$ & -0.0104 & 0.4897 & -1.0465 & 1.5346 & -0.2973 & 0.6320 & -0.6086 & 0.0477 & 0.5106 & 0.7812 & 0 \\
\hline $\mathrm{K}-\mathrm{I}$ & 0 & 0 & $v$ & $\frac{v(1-v)}{2}$ & 0 & $\frac{v}{8}$ & 0 & 0 & 0 & $\frac{v(1-v)}{16}$ & $\cdots$ \\
\hline K-I & 0 & 0 & $\frac{3}{5}$ & $\frac{3}{25}$ & 0 & $\frac{3}{40}$ & 0 & 0 & 0 & $\frac{3}{200}$ & $\ldots$ \\
\hline K-II & 0 & 0 & $\frac{3}{5}$ & $\frac{3}{25}$ & 0 & $\frac{3}{40}$ & 0 & 0 & 0 & $\frac{3}{200}$ & $\cdots$ \\
\hline \multicolumn{12}{|l|}{$B_{4}$} \\
\hline $\mathrm{BH}$ & -0.0008 & -0.0206 & 0.0728 & -0.0567 & 0.1925 & -0.3881 & 0.6402 & -0.6585 & 0.5542 & 1.8555 & 0 \\
\hline K-I & 0 & 0 & 0 & 0 & 0 & $v$ & 0 & 0 & 0 & $\frac{v(1-v)}{2}$ & $\ldots$ \\
\hline K-I & 0 & 0 & 0 & 0 & 0 & $\frac{3}{5}$ & 0 & 0 & 0 & $\frac{3}{25}^{2}$ & $\ldots$ \\
\hline K-II & 0 & 0 & 0 & 0 & 0 & 1 & 0 & 0 & 0 & 0 & 0 \\
\hline \multicolumn{12}{|l|}{$M_{4}$} \\
\hline $\mathrm{BH}$ & -0.0083 & 0.1693 & -0.5248 & 0.3997 & 0.5911 & -1.2406 & 1.7894 & -2.283 & 5.1783 & 3.3413 & 0 \\
\hline K-I & 0 & 0 & 0 & 0 & 0 & $v$ & 0 & $2 v$ & $v(1-v)$ & $\frac{v(1-v)}{2}$ & $\cdots$ \\
\hline K-I & 0 & 0 & 0 & 0 & 0 & $\frac{3}{5}$ & 0 & $\frac{6}{5}$ & $\frac{6}{25}$ & $\frac{6}{25}$ & $\ldots$ \\
\hline K-II & 0 & 0 & 0 & 0 & 0 & 1 & 0 & 2 & 0 & 0 & 0 \\
\hline
\end{tabular}

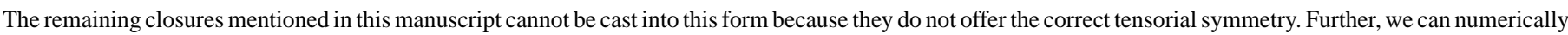

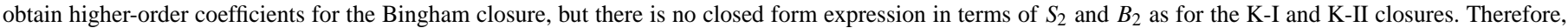

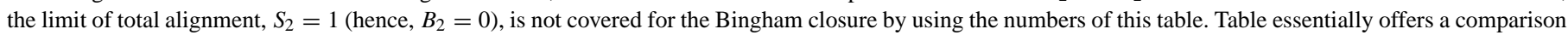

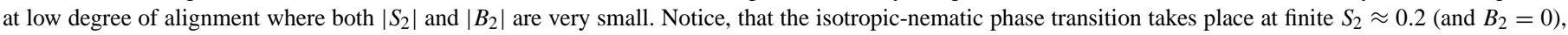
and $S_{2}$ may become much larger at lower temperatures, and in nonequilibrium situations, where the Taylor expansion is certainly not a useful approach. 

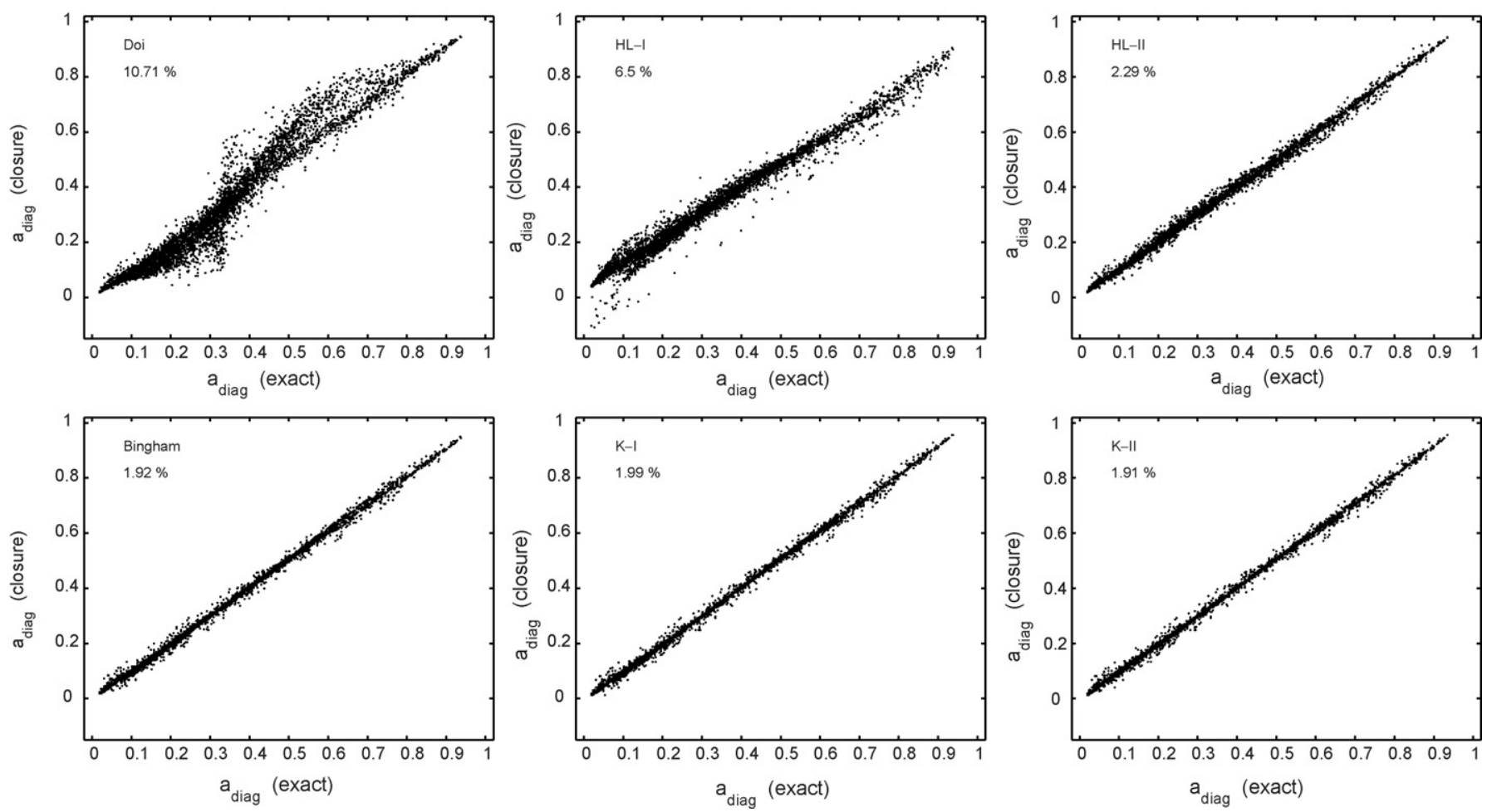

Fig. 4. Diagonal elements of the orientation tensor $\langle\boldsymbol{u} \boldsymbol{u}\rangle$ obtained using a closure approximation when solving the simple dynamical Eq. (35) vs. the "exact" (numerical) solution of the corresponding FP Eq. (61) for a very broad range of parameter values (2300 independent simulation runs) which cover biaxial, uniaxial, stationary, time-dependent states for randomly chosen particle geometries, flow geometries and flow strengths. The figures report errors for all the tested closure approximations: (a) Doi, (b) HL-I, (c) HL-II, (d) Bingham, (e) K-I, and (f) K-II, where the two latter ones have been proposed in this manuscript. Using a closure, the computational effort had been reduced by three orders of magnitude.
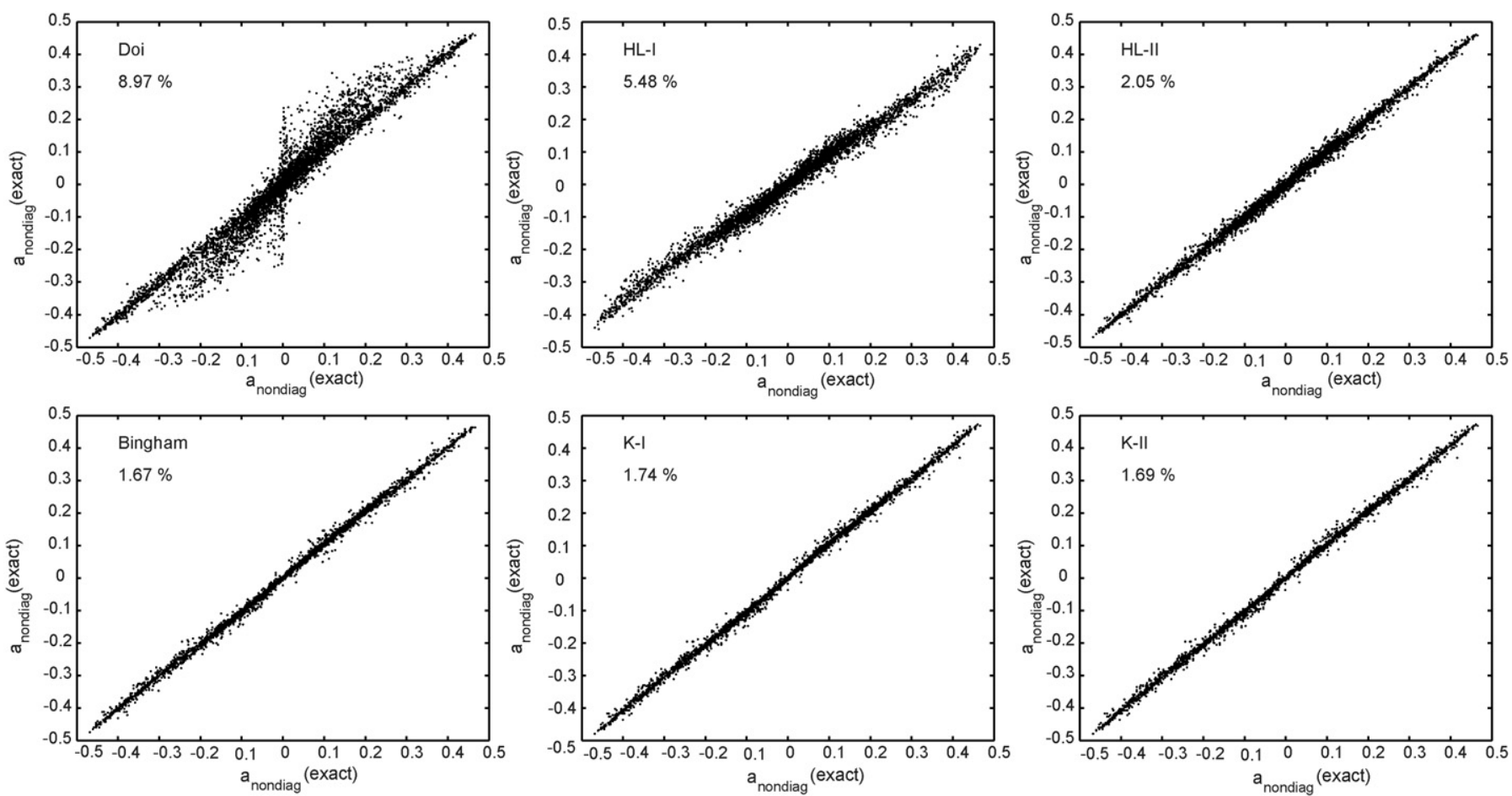

Fig. 5. Same as Fig. 4 for the nondiagonal components of the orientation tensor $\langle\boldsymbol{u} \boldsymbol{u}\rangle$. 


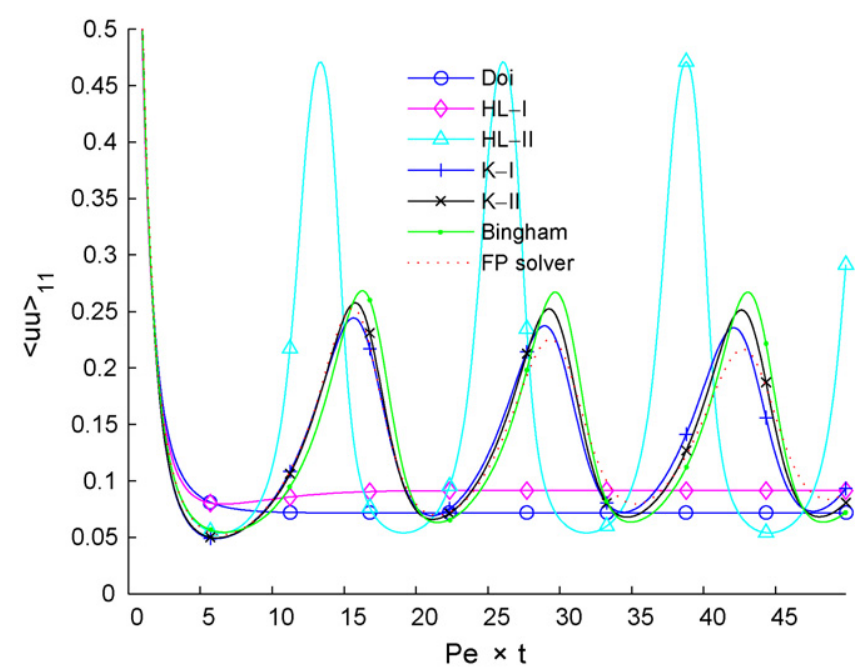

Fig. 6. Sample time series for alignment tensor component $\boldsymbol{a}_{(2) 11}=\langle\boldsymbol{u} \boldsymbol{u}\rangle_{11}$, with initial condition $\boldsymbol{a}_{(2)}(t=0)=\boldsymbol{e}_{1} \boldsymbol{e}_{1}$, strength $U=8$, flow field $\nabla \boldsymbol{v}=$ $\operatorname{Pe}\left[\boldsymbol{e}_{1} \boldsymbol{e}_{2}+\alpha \boldsymbol{e}_{2} \boldsymbol{e}_{1}\right], \alpha=0.05$. Comparison between closure approximations and numerical solution of the Hess-Doi FP equation.

defined in terms of Legendre polynomials and directors in (A.3) and (A.7) of Appendix A.

\section{Testing the biaxial closures}

As for Section 4.3.1, we consider the Hess-Doi FP equation for nematic and nematic-discotic liquid crystals [10,20]

$$
\begin{aligned}
\frac{\partial}{\partial t} f= & -\boldsymbol{\omega} \cdot \mathcal{L} f-\frac{1}{2} R \mathcal{L} \cdot f \mathcal{L} \boldsymbol{u}_{[2]}: \boldsymbol{D}+\frac{D_{\mathrm{r}}}{k_{\mathrm{B}} T} \mathcal{L} \cdot f \mathcal{L} \frac{\delta V}{\delta f} \\
& +D_{\mathrm{r}} \mathcal{L}^{2} f,
\end{aligned}
$$

with vorticity $\boldsymbol{\omega}=\frac{1}{2} \nabla \times \mathbf{v}, \boldsymbol{D}=\overrightarrow{\nabla \boldsymbol{v}}$, rotational operator $\mathcal{L} \equiv$ $\boldsymbol{u} \times \partial / \partial \boldsymbol{u}$, shape factor $R=\left(Q^{2}-1\right) /\left(Q^{2}+1\right)$ of an ellipsoid of revolution with axis ratio $Q=a / b$ [9], rotational diffusion coefficient $D_{\mathrm{r}}$, and a mean-field functional $V$ of the form $V[f]=-\frac{3}{2} U k_{\mathrm{B}} T \boldsymbol{a}_{[2]}: \boldsymbol{a}_{[2]}$. The FP equation has been extended

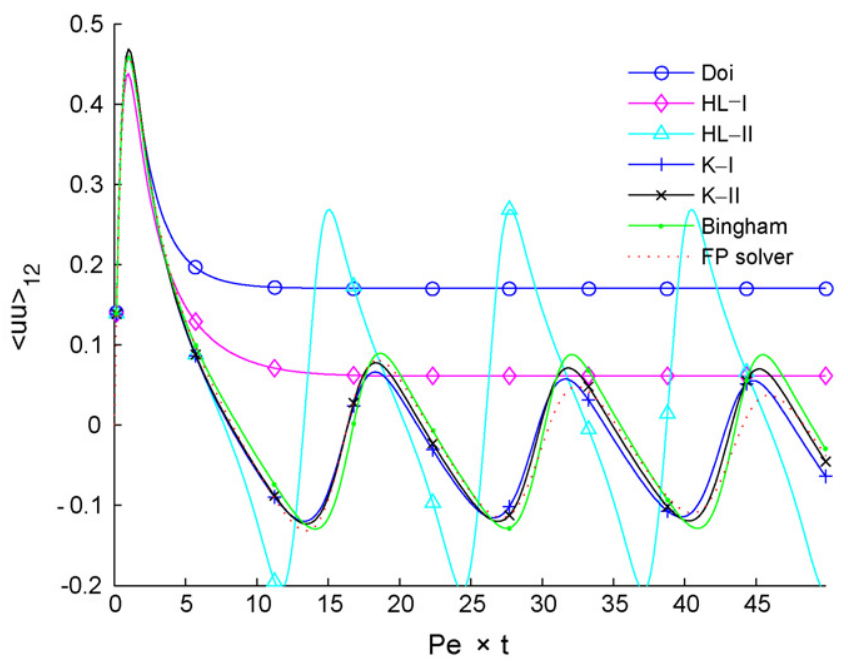

Fig. 7. Same as Fig. 6 for a nondiagonal component of the alignment tensor. to non-uniform nematics in [45]. The equation of change for the alignment tensor derived from (61) was already stated above, cf. Eq. (35). The closed version of it is immediately obtained by using the identities (B.2) and (B.4) together with a consistent closure (K-I, K-II, Bingham, etc., cf. Table 4). It can be conveniently solved using a basic solver for ordinary differential equations. On the other hand, we solve the FP equation using a reduction method as described in Appendix C. By largely varying the parameters of the FP equation this approach sets us in the position to rigorously test the closure approximation. Specifically, we performed simulations for dimensionless model parameters equally and randomly distributed within the following bounds: $-1 \leq R \leq 1,0.2 \leq D_{\mathrm{r}} \leq 5,0 \leq U \leq 20,-1 \leq$ $(\nabla \mathbf{v})_{\mu v} \leq 1$ where the latter holds for all components except for the constrained component $(\nabla \mathbf{v})_{33}=-(\nabla \mathbf{v})_{11}-(\nabla \mathbf{v})_{22}$ in order to study incompressible flows. The results are collected in Figs. 4-7 . They remain qualitatively unaltered if we add a potential $V$ corresponding to an orienting magnetic field. For this reason, there is no need to report about these additional results here.

\section{Summary}

We propose the 'quadratic' closure scheme (K-I)

$\forall_{n, m} \boldsymbol{a}_{[n+m]}=\zeta \overline{\boldsymbol{a}_{[n]} \boldsymbol{a}_{[m]}}$,

where $\ldots$ denotes the symmetric traceless (irreducible) part, as a more rational approach to approximating the various (symmetric traceless) alignment tensors $\boldsymbol{a}_{[n]}$ of rank $n$. Eq. (62) with $\zeta=1$ (quadratic III), or $\zeta=S_{n+m} /\left(S_{n} S_{m}\right)$ (K-I) obtained with the equilibrium distribution function are the simplest closures with correct tensorial symmetry, they are correct in both the isotropic and totally aligned phases. Of uppermost relevance for applications is the case $n=m=2$ in (62), while for the implementation of the higher-order decoupling approximations the representation theorems in Section 10.4.1 of [9] can be used. In order to easily apply the new closures, summarized in Section 5 of this manuscript, we introduced order parameters and established identities to be used when writing down the decoupled equation of change for the alignment tensor. Worked out explicit closure relationships for the uniaxial phase, parameterized by a coefficient $v>0$ have been given for the fourth moment in terms of the second one in (16) with (21) and (25), and compared to 'inconsistent' closures in Tables 2 and 3. Exact closures for the second and third moments in terms of the first moment are given in (43). The closure (62) has been worked out for the biaxial case (and $n=m=2$ ) in Section 5 and tested for the case of the Hess-Doi FP equation for liquid crystals in Section 6. In [12,28], several closure schemes had been compared for the dynamics of liquid crystals in an exhaustive fashion. It remains to be seen, how these comparisons alter if the closures K-I (57), (B.1), K-II (60), (B.1) with correct tensorial symmetry are used. It is known that the Bingham distribution $f(\boldsymbol{u}) \propto \exp \left(\propto \boldsymbol{A}: \boldsymbol{u}_{(2)}\right)$, which is the biaxial analogue to the Maier-Saupe distribution $f(\boldsymbol{u}) \propto \exp \left\{\propto P_{2}(\boldsymbol{u} \cdot \boldsymbol{n})\right\}$, performs very well when considering the flow behavior [28] and 
nonequilibrium phase diagram $[15,16,46]$ of liquid crystalline polymers, certainly, because assuming a distribution function of this type leads to closure relationships which are special cases of ones presented here, and thus, in opposite to alternate closures, take into account tensorial symmetries. This manuscript aimed at illustrating these ideas from a different perspective, proposes new and simple closures shown to be useful in the uniaxial and biaxial phases, and also extends the arguments to tensors of arbitrary rank.

\section{Acknowledgments}

The author would like to thank Patrick Ilg for helpful discussions. This work has been supported by NSF-EC FP6-016375 (MNIBS) and EU Priority FP6-2004-NMP-TI-4 STRP 033339 related grants of the European Community.

\section{Appendix A. Biaxial phase}

In the biaxial phase, the three principal values of (8) are distinct. The methods described in Section 4 for the uniaxial phase can be immediately taken over to the biaxial phase. Instead of a single scalar order parameter $S_{2}$ for the second moment, and a single scalar order parameter $S_{4}$ for the fourth moment, we have to deal with two scalar order parameters $S_{2}, B_{2}$ for the second moment, and three scalar order parameters $S_{4}, B_{4}, M_{4}$ (which depend on $S_{2}, B_{2}$ ) for the fourth moment, because these tensors share the same eigenvectors. All rules needed for handling the biaxial case are most easily obtained by introducing orthogonal unit vectors $\boldsymbol{e}_{1}=\boldsymbol{n}$ and $\boldsymbol{e}_{2}=$ $\boldsymbol{m}$ with $\boldsymbol{e}_{i}=\left(\cos \phi_{i} \sin \theta_{i}, \sin \phi_{i} \sin \theta_{i}, \cos \theta_{i}\right)$ and the coupling $\theta_{1}=-\operatorname{arccot}\left[\cos \left(\phi_{1}-\phi_{2}\right) \tan \theta_{2}\right]$ ensuring $|\boldsymbol{n}|=|\boldsymbol{m}|=1$ and $\boldsymbol{n} \cdot \boldsymbol{m}=0$ by construction. The set of orthonormal eigenvectors is completed by a third unit vector $\boldsymbol{l}$ via $\boldsymbol{n}_{(2)}+\boldsymbol{m}_{(2)}+\boldsymbol{l}_{(2)}=\boldsymbol{I}$. Taking into account the constraint on the $\lambda_{i}$, we obtain

$$
\begin{aligned}
\boldsymbol{a}_{(2)}= & \sum_{i} \lambda_{i} \boldsymbol{e}_{i} \boldsymbol{e}_{i}=\frac{1}{3}\left(2 S_{2}+1\right) \boldsymbol{n}_{(2)}+\frac{1}{3}\left(\frac{3}{2} B_{2}-S_{2}+1\right) \boldsymbol{m}_{(2)} \\
& -\frac{1}{3}\left(\frac{3}{2} B_{2}+S_{2}-1\right) \boldsymbol{l}_{(2)},
\end{aligned}
$$

or equivalently, in a more compact fashion, illuminating the role of $B_{2}$,

$\boldsymbol{a}_{[2]}=\boldsymbol{a}_{(2)}-\frac{1}{3} \boldsymbol{I}=\left(S_{2}+B_{2} / 2\right) \boldsymbol{n}_{[2]}+B_{2} \boldsymbol{m}_{[2]}$,

where $\boldsymbol{n}_{[2]}=\overline{\boldsymbol{n}_{(2)}}=\boldsymbol{n}_{(2)}-\frac{1}{3} \boldsymbol{I}, B_{2}$ is the second-order scalar biaxial order parameter

$$
B_{2}=\frac{2}{3}\left(\left\langle P_{2}(\boldsymbol{u} \cdot \boldsymbol{n})\right\rangle+2\left\langle P_{2}(\boldsymbol{u} \cdot \boldsymbol{m})\right\rangle\right)=\left\langle(\boldsymbol{u} \cdot \boldsymbol{m})^{2}-(\boldsymbol{u} \cdot \boldsymbol{l})^{2}\right\rangle,
$$

and we have, for example, $\frac{3}{2} \boldsymbol{a}_{[2]}: \boldsymbol{a}_{[2]}=S_{2}^{2}+\frac{3}{4} B_{2}^{2}$ or $\operatorname{tr}\left(\boldsymbol{a}_{[2]}\right.$. $\left.\boldsymbol{a}_{[2]} \cdot \boldsymbol{a}_{[2]}\right)=S_{2}^{3}+\frac{3}{2} S_{2} B_{2}\left(S_{2}+\frac{B_{2}}{2}\right)+\frac{9}{8} B_{2}^{3}$. If we again identify $\boldsymbol{n}=\boldsymbol{e}_{1}, \boldsymbol{m}=\boldsymbol{e}_{2}$, and $l=\boldsymbol{e}_{3}$, then $S_{2}=\frac{3}{2}\left\langle x^{2}\right\rangle-\frac{1}{2}$ and $B_{2}=\left\langle y^{2}-z^{2}\right\rangle$. Upon applying $\lambda_{n}>\lambda_{m}>\lambda_{l}$ to define $\boldsymbol{n}, \boldsymbol{m}$, and $\boldsymbol{l}, S_{2}$ is semipositive, and $B_{2}$ ranges in value by $0 \leq B_{2} \leq$ $2 S_{2} \leq \frac{1}{2}$ and $0 \leq B_{2} \leq \frac{2}{3}\left(1-S_{2}\right) \leq \frac{1}{2}$ for $S_{2} \leq \frac{1}{4}$ and $S_{2} \geq \frac{1}{4}$, respectively. For perfect uniaxial alignment in the $\boldsymbol{n}$ direction, $S_{2}=1$ and $B_{2}=0$. For random alignment (hence, isotropic) $S_{2}=B_{2}=0$. Similarly, we obtain for the fourth-order alignment tensors

$$
\begin{aligned}
\boldsymbol{a}_{(4)}= & \left(S_{4}-\frac{3}{8} B_{4}+\frac{1}{2} M_{4}\right) \boldsymbol{n}_{(4)}+B_{4} \boldsymbol{m}_{(4)}+M_{4}\left\{\boldsymbol{n}_{(2)} \boldsymbol{m}_{(2)}\right\}_{\mathrm{sym}} \\
& +\alpha_{1}\left\{\boldsymbol{n}_{(2)} \boldsymbol{I}\right\}_{\mathrm{sym}}+\alpha_{2}\left\{\boldsymbol{m}_{(2)} \boldsymbol{I}\right\}_{\mathrm{sym}}+\alpha_{3}\{\boldsymbol{I} \boldsymbol{I}\}_{\mathrm{sym}}
\end{aligned}
$$

$\boldsymbol{a}_{[4]}=\left(S_{4}-\frac{3}{8} B_{4}+\frac{1}{2} M_{4}\right) \boldsymbol{n}_{[4]}+B_{4} \boldsymbol{m}_{(4)}+M_{4} \overline{\boldsymbol{n}_{(2)} \boldsymbol{m}_{(2)}}$,

with

$$
\begin{aligned}
& \alpha_{1}=\frac{1}{28}\left[24\left(S_{2}-S_{4}\right)+12 B_{2}+9 B_{4}-16 M_{4}\right], \\
& \alpha_{2}=\frac{1}{7}\left[6\left(B_{2}-B_{4}\right)-M_{4}\right], \\
& \alpha_{3}=\frac{1}{280}\left[4\left(14+5 M_{4}-20 S_{2}+6 S_{4}\right)-120 B_{2}+15 B_{4}\right],
\end{aligned}
$$

where

$$
\begin{aligned}
& S_{4}=\left\langle P_{4}(\boldsymbol{n} \cdot \boldsymbol{u})\right\rangle, \\
& B_{4}=\frac{8}{35}\left[4\left\langle P_{4}(\boldsymbol{m} \cdot \boldsymbol{u})\right\rangle+4\left\langle P_{4}(\boldsymbol{l} \cdot \boldsymbol{u})\right\rangle-3\left\langle P_{4}(\boldsymbol{n} \cdot \boldsymbol{u})\right\rangle\right], \\
& M_{4}=\frac{8}{35}\left[11\left\langle P_{4}(\boldsymbol{l} \cdot \boldsymbol{u})\right\rangle-3\left\langle P_{4}(\boldsymbol{n} \cdot \boldsymbol{u})\right\rangle-3\left\langle P_{4}(\boldsymbol{m} \cdot \boldsymbol{u})\right\rangle\right] .
\end{aligned}
$$

Note that there are three distinct fourth-order scalar measures of alignment: $S_{4}, B_{4}$, and $M_{4}$. In the uniaxial case with director $\boldsymbol{n}$, we have $B_{4}=M_{4}=0$, so that these two can be interpreted as fourth-order measures of the deviation from uniaxiality.

Any closure relationship for the biaxial phase with correct tensorial symmetry is therefore equivalent with a set of three functions $S_{4}, B_{4}$, and $M_{4}$ in terms of the order parameters $\left(S_{2}, B_{2}\right)$. The three functions are subject to several boundary conditions such as $S_{4}(1,0)=1, S_{4}(0,0)=0, M_{4}(\cdot, 0)=0$, $B_{4}(\cdot, 0)=0$ and their domain is restricted according to $0 \leq$ $B_{2} \leq \frac{2}{3}\left(1-S_{2}\right) \leq \frac{1}{2}$ for $S_{2} \leq \frac{1}{4}$ and $S_{2} \geq \frac{1}{4}$, respectively. At fist glance, it does not seem possible to express the fourth-order moments $\boldsymbol{a}_{(4)}$ and $\boldsymbol{a}_{[4]}$ directly in terms of the second-order moments $\boldsymbol{a}_{(2)}$ and $\boldsymbol{a}_{[2]}$ without some type of approximation (e.g., $M_{4}=0$ ), as was possible in the uniaxial and isotropic cases. However, the orthonormal directors $\boldsymbol{n}$ and $\boldsymbol{m}$ as well as the eigenvalues of $\boldsymbol{a}_{[2]}$, or equivalently, order parameters $S_{2}$ and $B_{2}$ can be calculated using straightforward calculus. With these quantities at hand, we can directly insert into (A.5) to obtain the desired relationship between $\boldsymbol{a}_{[4]}$ and $\boldsymbol{a}_{[2]}$ (which will be of the form presented in [25], where we have imposed restrictions to the coefficients), and also an expressions for the biaxial generalization of (25). Moreover, concerning applications, we usually need closure relationships for second rank tensors like $\boldsymbol{X}$ or $\boldsymbol{a}_{[4]}: \boldsymbol{a}_{[2]}$ (actually, both tensors being symmetric traceless), which must 
be, due to the Caley-Hamilton theorem, expressable as linear combination of $\boldsymbol{a}_{[2]}$ and $\boldsymbol{a}_{[2]} \cdot \boldsymbol{a}_{[2]}[9]$.

\section{Appendix B. Identities valid for the biaxial phase}

In order to present more useful expressions for the most general closure (53) for biaxial liquid crystals, including all consistent closures in a unifying fashion, let us write down explicit expressions for the quantities $\boldsymbol{D}: \boldsymbol{a}_{(4)}$ and $\boldsymbol{X}$ appearing in (35). The former term with symmetric traceless 2 nd rank tensor $\boldsymbol{D}$ and $\boldsymbol{a}_{(4)}=\langle\boldsymbol{u} \boldsymbol{u} \boldsymbol{u} \boldsymbol{u}\rangle$ reads, using (52) and (53)

$$
\begin{aligned}
\boldsymbol{D}: \boldsymbol{a}_{(4)}= & D_{1} \boldsymbol{D}+D_{2} \boldsymbol{D}: \boldsymbol{n n}+D_{3} \boldsymbol{D}: \boldsymbol{m} \boldsymbol{m} \\
& +D_{4}(\boldsymbol{n} \boldsymbol{n} \cdot \boldsymbol{D}+\boldsymbol{D} \cdot \boldsymbol{n n})+D_{5}(\boldsymbol{m} \boldsymbol{m} \cdot \boldsymbol{D}+\boldsymbol{D} \cdot \boldsymbol{m} \boldsymbol{m}) \\
& +D_{6}(\boldsymbol{m} \cdot \boldsymbol{D} \cdot \boldsymbol{n})(\boldsymbol{n} \boldsymbol{m}+\boldsymbol{m} \boldsymbol{n}),
\end{aligned}
$$

with

$$
\begin{aligned}
D_{1}= & \frac{2}{15}-\frac{4 S_{2}}{21}+\frac{2 S_{4}}{35}-\frac{2 B_{2}}{7}+\frac{B_{4}}{28}+\frac{M_{4}}{21}, \\
D_{2}= & \frac{1}{7}\left(S_{2}+\frac{4 S_{4}}{3}+\frac{B_{2}}{2}-\frac{B_{4}}{2}+\frac{8 M_{4}}{9}\right) \boldsymbol{I} \\
& +\left(S_{4}-\frac{3 B_{4}}{8}+\frac{M_{4}}{2}\right) \boldsymbol{n}_{[2]}+\frac{M_{4}}{6} \boldsymbol{m}_{[2]}, \\
D_{3}= & \frac{1}{7}\left(B_{2}+\frac{4 B_{4}}{3}+\frac{2 M_{4}}{9}\right) \boldsymbol{I}+\frac{M_{4}}{6} \boldsymbol{n}_{[2]}+B_{4} \boldsymbol{m}_{[2]}, \\
D_{4}= & \frac{2}{7}\left(S_{2}-S_{4}+\frac{B_{2}}{2}+\frac{3 B_{4}}{8}-\frac{2 M_{4}}{3}\right), \\
D_{5}= & \frac{2}{7}\left(B_{2}-B_{4}-\frac{M_{4}}{6}\right), D_{6}=\frac{M_{4}}{3},
\end{aligned}
$$

where $\boldsymbol{n}_{[2]}$ and $\boldsymbol{m}_{[2]}$ are defined above. We have therefore expressed $\boldsymbol{D}: \boldsymbol{a}_{(2)}$ in terms of $\boldsymbol{a}_{(2)}$ and three unspecified order parameters $S_{4}, B_{4}$, and $M_{4}$. The identify is of course still valid in the uniaxial phase, where $B_{2}=B_{4}=M_{4}=0$.

Using identity (B.2) for the special case $\boldsymbol{D}=\boldsymbol{a}_{[2]}$, the traceless quantity $\boldsymbol{X} \equiv \boldsymbol{a}_{(2)} \cdot \boldsymbol{a}_{(2)}-\boldsymbol{a}_{(4)}: \boldsymbol{a}_{(2)}$ with $\boldsymbol{a}_{(2)} \equiv\langle\boldsymbol{u} \boldsymbol{u}\rangle$ and $\boldsymbol{a}_{(4)} \equiv\langle\boldsymbol{u} \boldsymbol{u} \boldsymbol{u} \boldsymbol{u}\rangle$ becomes

$\boldsymbol{X}=X_{n} \boldsymbol{n}_{[2]}+X_{m} \boldsymbol{m}_{[2]}$,

with

$$
\begin{aligned}
X_{n}= & S_{2}\left(\frac{1}{5}+\frac{S_{2}}{7}-\frac{B_{2}}{7}-\frac{12 S_{4}}{35}+\frac{B_{4}}{14}-\frac{M_{4}}{14}\right) \\
& +B_{2}\left(\frac{1}{10}-\frac{3 B_{2}}{28}-\frac{S_{4}}{35}-\frac{B_{4}}{56}-\frac{3 M_{4}}{28}\right), \\
X_{m}= & S_{2}\left(-\frac{2 B_{2}}{7}+\frac{B_{4}}{7}-\frac{M_{4}}{7}\right)+B_{2}\left(\frac{1}{5}-\frac{B_{4}}{4}-\frac{2 S_{4}}{35}\right) .
\end{aligned}
$$

We have therefore expressed $\boldsymbol{X}$ in terms of $\boldsymbol{a}_{(2)}$ and three unspecified order parameters $S_{4}, B_{4}$, and $M_{4}$.

Upon inserting (B.3), (B.1) with (B.4), (B.2) into an equation of change such as (35) we are left with a parameter-free closed dynamical equation for the orientation tensor $\boldsymbol{a}_{(2)}$, once we have suitable expressions for the order parameters $S_{4}, B_{4}, M_{4}$ in terms of $S_{2}$ and $B_{2}$. The term (B.1) considerably simplifies if one is interested in a particular flow geometry only such as shear flow with shear rate $\dot{\gamma}$ for which $(\nabla \mathbf{v})_{\mu \nu}=\dot{\gamma} \delta_{1, \mu} \delta_{2, \nu}$ and therefore $(\boldsymbol{D})_{\mu \nu}=\frac{1}{2} \dot{\gamma}\left(\delta_{2, \mu} \delta_{1, \nu}+\delta_{1, \mu} \delta_{2, \nu}\right)$ and $\boldsymbol{W}$ equals $\boldsymbol{D}$ upon replacing ' + ' by ' - '. If a magnetic field $\mathbf{H}$ is present, the equation of change (35) has to be modified in such a way that $\boldsymbol{D}$ is replaced by $\tilde{\boldsymbol{D}}$ with (symmetric traceless) $\tilde{\boldsymbol{D}} \equiv \boldsymbol{D}+D_{\mathrm{r}} R^{-1} \chi_{a} \mathbf{H H} / k_{\mathrm{B}} T$, anisotropic magnetic susceptibility $\chi_{a}=\chi_{\|}-\chi_{\perp}$, and $\mathbf{H H}=$ HH $-\frac{1}{3} H^{2} \boldsymbol{I}[9,20]$.

\section{Appendix C. Reduced order modelling}

A large variety of kinetic theory models related to complex fluids can be described from a parabolic partial differential equation, such as the FP Eq. (61), governing the evolution in time of the fluid microstructure conformation. When one is solving a non-linear parabolic partial differential equation using some standard technique, as for example, the finite element method, at least a linear system must be solved at each time step. The size of this system coincides with the number of degrees of freedom used to describe the space evolution of the considered field. In kinetic theory models the space coordinates group the physical and the conformational ones.

It has been widely noticed in a large variety of models involving linear and non-linear parabolic partial differential equations that the field evolution in the whole time interval can be accurately represented from the linear combination of a reduced number of functions that are defined in the whole spatial domain. In that follows we are illustrating the consequences of this behavior.

We assume that the evolution of a certain field $\Psi(\mathbf{x}, t)$ is known. In practical applications, this field is expressed in a discrete form, that is, it is known at the $N_{n}$ nodes of a spatial mesh and for some times $\Psi^{n}(\mathbf{x})=\Psi(\mathbf{x}, t=n \Delta t), \forall n \in[1, \ldots, P]$. The main idea of the Karhunen-Love (KL) decomposition is how to obtain the most typical or characteristic structure $\phi(\mathbf{x})$ among these $\Psi^{n}(\mathbf{x}), \forall n$. This is equivalent of obtaining a function $\phi(\mathbf{x})$ that maximizes $\lambda$ defined by

$$
\lambda=\frac{\sum_{n=1}^{n=P}\left[\sum_{i=1}^{i=N_{n}} \phi\left(\mathbf{x}_{i}\right) \Psi^{n}\left(\mathbf{x}_{i}\right)\right]^{2}}{\sum_{i=1}^{i=N}\left(\phi\left(\mathbf{x}_{i}\right)\right)^{2}} .
$$

The maximization $(\delta \lambda=0)$ leads to the following eigenproblem

$\left(\mathbf{Q} \cdot \mathbf{Q}^{T}\right) \cdot \boldsymbol{\Phi}=\lambda \boldsymbol{\Phi}$, 
where $\boldsymbol{\Phi}$ represents the vector containing the nodal values of function $\phi$ and the matrix $\mathbf{Q}$ contains the discrete field history:

$$
\mathbf{Q}=\left(\begin{array}{cccc}
\Psi_{1}^{1} & \Psi_{1}^{2} & \cdots & \Psi_{1}^{P} \\
\Psi_{2}^{1} & \Psi_{2}^{2} & \cdots & \Psi_{2}^{P} \\
\vdots & \vdots & \ddots & \vdots \\
\Psi_{N_{n}}^{1} & \Psi_{N_{n}}^{2} & \cdots & \Psi_{N_{n}}^{P}
\end{array}\right)
$$

The solution of the eigenproblem (C.2) results in $N_{n}$ eigenvalue-eigenvector pairs: $\left(\Phi_{k}, \lambda_{k}\right), k \in\left[1, \ldots, N_{n}\right]$. As just argued, in numerous problems whose solutions are characterized by a regularity in their time evolutions, the most part of the eigenvalues are small compared with large ones. In fact, the proper orthogonal KL decomposition establishes that the magnitude of the eigenvalues represents the intensity with which the corresponding eigenvector is present in the solution representation. Crudely speaking, one can neglect in the approximation of the field the eigenvectors related to the eigenvalues verifying the relation $\lambda_{k}<\epsilon \lambda_{1}$, where $\epsilon$ is a small enough parameter ( $\epsilon=10^{-8}$ in our simulations) and $\lambda_{1}$ denotes the highest eigenvalue. Thus, if only $r$ eigenvalues are retained, one can expect that the evolution of the field $\Psi$ can be expressed from the associated eigenvectors $\boldsymbol{\Phi}_{k}, k \in[1, \ldots, r]$ :

$\boldsymbol{\Psi}(t)=\sum_{k=1}^{k=r} a_{k}(t) \boldsymbol{\Phi}_{k}$,

where vector $\Psi(t)$ contains the nodal values of function $\Psi$ at time $t$. Thus, we define the matrix allowing the transformation between the usual finite element approximation basis and the reduced one:

$\mathbf{B}=\left(\begin{array}{cccc}\phi_{1}\left(\mathbf{x}_{1}\right) & \phi_{2}\left(\mathbf{x}_{1}\right) & \cdots & \phi_{r}\left(\mathbf{x}_{1}\right) \\ \phi_{1}\left(\mathbf{x}_{2}\right) & \phi_{2}\left(\mathbf{x}_{2}\right) & \ldots & \phi_{r}\left(\mathbf{x}_{2}\right) \\ \vdots & \vdots & \ddots & \vdots \\ \phi_{1}\left(\mathbf{x}_{N_{n}}\right) & \phi_{2}\left(\mathbf{x}_{N_{n}}\right) & \cdots & \phi_{r}\left(\mathbf{x}_{N_{n}}\right)\end{array}\right)$.

Now, we consider the linear system of equations resulting from the discretisation of the FP equation when an explicit time discretization scheme is used:

$\mathbf{K} \cdot \Psi^{n}=\mathbf{F}^{n-1}$,

where $\mathbf{F}^{n-1}$ contains the contribution of the solution at the previous time step.

Then, the unknown vector containing the nodal degrees of freedom can be expressed as

$\boldsymbol{\Psi}^{n}=\sum_{i=1}^{i=r} \boldsymbol{\Phi}_{i} a_{i}^{n}=\mathbf{B} \cdot \mathbf{a}^{n}$,

which implies

$\mathbf{K} \cdot \boldsymbol{\Psi}^{n}=\mathbf{F}^{n-1} \Rightarrow \mathbf{K} \cdot \mathbf{B} \cdot \mathbf{a}^{n}=\mathbf{F}^{n-1}$,

and multiplying both terms by $\mathbf{B}^{T}$ it results

$\mathbf{B}^{T} \cdot \mathbf{K} \cdot \mathbf{B} \cdot \mathbf{a}^{n}=\mathbf{B}^{T} \cdot \mathbf{F}^{n-1}$, which proves that the final linear system is of small size, i.e. the dimensions of $\mathbf{B}^{T} \cdot \mathbf{K} \cdot \mathbf{B}$ are $r \times r$, with $r \ll N_{n}$, and the dimensions of both $\mathbf{a}$ and $\mathbf{B}^{T} \cdot \mathbf{F}$ are $r \times 1$. Eq. (C.9) can be also derived introducing the approximation (C.7) into the PDE Galerkin form.

In the previous paragraphs we have illustrated the procedure to extract the significant functions representing the evolution of the field of interest in the whole time interval when this evolution is known, as a simple application of the proper orthogonal decomposition. These characteristic functions are then used to solve accurately the evolution problem, which in this case only implies a reduced number of degrees of freedom, and consequently a significant reduction of the computing time is expected. However, the procedure is not operative because in order to define the reduced basis the solution must be previously computed.

In [47], we have proposed an adaptive procedure in order to define the reduced approximation basis during the solution and without any knowledge a priori. We are summarizing the main ideas of the solution procedure. For more details the reader can refer the just referred paper. The solution procedure start by assuming that matrix $\mathbf{B}$ contains a single arbitrary function that could consist in the initial condition. Now, the solution is computed in the entire time interval, from which the residual $\mathbf{R}$ is computed at time $t=P \Delta t$ :

$\mathbf{R}=\mathbf{K} \cdot \mathbf{B} \cdot \mathbf{a}^{P}-\mathbf{F}^{P-1}$

If the residual norm is greater than a threshold value, the reduced basis is enriched using the residual $\mathbf{B} \leftarrow(\mathbf{B}, \mathbf{R})$ and the solution reevaluated in the entire time interval. This iteration procedure continues until convergence. We have proved that this algorithm is robust, efficient (it converges in few iterations) and that it allows a significant CPU time reduction. It has been used to solve the FP equation in Section 6.

\section{References}

[1] F.M. Leslie, Some constitutive equations for anisotropic fluids, Quart. J. Mech. Appl. Math. 19 (1966) 357.

[2] F.M. Leslie, Some constitutive equations for liquid crystals, Arch. Ration. Mech. Anal. 28 (1968) 265.

[3] F.M. Leslie, Continuum theory for nematic liquid crystals, Cont. Mech. Thermodyn. 4 (1992) 167.

[4] F.M. Leslie, J.S. Laverty, T. Carlsson, Continuum theory for biaxial nematic liquid crystals, Q. J. Mech. Appl. Math. 45 (1992) 595.

[5] P. Ilg, M. Kröger, Magnetization dynamics, rheology, and an effective description of ferromagnetic units in dilute suspension, Phys. Rev. E 66 (2002) 021501

[6] M. Kröger, Magnetoviscous model fluids, J. Phys.: Condens. Matter 15 (2003) S1403.

[7] Z.W. Wang, C. Holm, H.W. Muller, Molecular dynamics study on the equilibrium magnetization properties and structure of ferrofluids, Phys. Rev. E 66 (2002) 021405.

[8] M. Parsheh, M.L. Brown, C.K. Aidun, Cluster size distribution and scaling for spherical particles and red blood cells in pressure-driven flows at small Reynolds number, J. Non-Newt. Fluid Mech. 136 (2006) 38.

[9] M. Kröger, Models for polymeric and anisotropic liquids, Springer, Berlin, 2005.

[10] S. Hess, Fokker-Planck-equation approach to flow-alignment in liquid crystals, Z. Naturforsch. 31a (1976) 1034. 
[11] M. Doi, Molecular dynamics and rheological properties of concentrated solutions of rodlike polymers in isotropic and liquid crystalline phases, J. Polym. Sci., Polym. Phys. Ed. 19 (1981) 229-243.

[12] C.V. Chaubal, L.G. Leal, G.H. Fredrickson, A comparison of closure approximations for the Doi theory of LCPs, J. Rheol. 39 (1995) 73.

[13] R.G. Larson, H.C. Öttinger, Effect of molecular elasticity on out-of-plane orientations in shearing flows of liquid-crystalline polymers, Macromolecules 24 (1991) 6270.

[14] M. Kröger, Simple models for complex nonequilibrium fluids, Phys. Rep. 390 (2004) 453.

[15] G. Rienäcker, M. Kröger, S. Hess, Chaotic orientational behavior of a nematic liquid crystal subjected to a steady shear flow, Phys. Rev. E 66 (2002) 040702.

[16] G. Rienäcker, M. Kröger, S. Hess, Chaotic and regular shear-induced orientational dynamics of nematic liquid crystals, Physica A 315 (2002) 537.

[17] S.M. Fielding, P.D. Olmsted, Spatiotemporal oscillations and rheochaos in a simple model of shear banding, Phys. Rev. Lett. 92 (2004) 084502.

[18] M.G. Forest, Q. Wang, R.H. Zhou, The weak shear kinetic phase diagram for nematic polymers, Rheol. Acta 43 (2004) 17.

[19] G.L. Hand, A theory of anisotropic fluids, J. Fluid Mech. 13 (1962) 33.

[20] M. Doi, S.F. Edwards, The Theory of Polymer Dynamics, Clarendon, Oxford, 1986.

[21] E.J. Hinch, L.G. Leal, Constitutive equations in suspension mechanics. Part 2. Approximate forms for a suspension of rigid particles affected by Brownian rotations, J. Fluid Mech. 76 (1976) 187.

[22] H. Ehrentraut, S. Hess, On the viscosity of partially aligned nematic and nematic discotic liquid crystals, Phys. Rev. E 51 (1995) 2203.

[23] M. Kröger, Viscoelasticity of polymeric melts and concentrated solutions. The effect of flow-induced alignment of chain ends, Physica A 195 (1993) 336.

[24] M. van Gurp, Letter to the Editor: on the use of spherical tensors and the maximum entropy method to obtain closure for anisotropic liquids, J. Rheol. 42 (1998) 1269.

[25] B.J. Edwards, H.C. Öttinger, Time-structure invariance criteria for closure approximations, Phys. Rev. E 56 (1997) 4097.

[26] H.C. Öttinger, Beyond Equilibrium Thermodynamics, Wiley \& Sons, Hoboken, NJ, 2005.

[27] C.V. Chaubal, L.G. Leal, A closure approximation for liquid-crystalline polymer models based on parametric density estimation, J. Rheol. 42 (1998) 177.

[28] J. Feng, C.V. Chaubal, L.G. Leal, Closure approximations for the Doi theory: which to use in simulating complex flows of liquid-crystalline polymers? J. Rheol. 42 (1998) 1095.

[29] R.H. Zhou, M.G. Forest, Q. Wang, Kinetic structure simulations of nematic polymers in plane Couette cells. I: the algorithm and benchmarks, Multiscale Model. Simul. 3 (2005) 853.
[30] J.N. Fang, R.G. Owens, New constitutive equations derived from a kinetic model for melts and concentrated solutions of linear polymers, Rheol. Acta 44 (2005) 577.

[31] P. Yu, Q. Du, C. Liu, From micro to macro dynamics via a new closure approximation to the FENE model of polymeric fluids, Multiscale Model. Simul. 3 (2005) 895.

[32] J.S. Cintra, C.L. Tucker, Orthotropic closure approximations for flowinduced fiber orientation, J. Rheol. 39 (1995) 1095.

[33] A.V. Bhave, R.K. Menon, R.C. Armstrong, R.A. Brown, A constitutive equation for liquid-crystalline polymer solutions, J. Rheol. 37 (1993) 413.

[34] S. Advani, C.L. Tucker, Closure approximations for three-dimensional structure tensors, J. Rheol. 34 (1990) 367.

[35] S. Hess, W. Köhler, Formeln der Tensorrechnung, Palm \& Enke, Erlangen, 1982.

[36] R.B. Bird, O. Hassager, R.C. Armstrong, C.F. Curtiss, Dynamics of Polymeric Liquids, vol. 1+2, Wiley \& Sons, NY, 1987.

[37] H.-H. Kyeong, T.-I. Yong, Modified hybrid closure approximation for prediction of flow-induced fiber orientation, J. Rheol. 43 (1999) 569.

[38] M. Kröger, H.S. Sellers, Viscosity coefficients for anisotropic, nematic fluids based on structural theories of suspensions, J. Chem. Phys. 103 (1995) 807.

[39] M. Kröger, W. Loose, S. Hess, Structural changes and rheology of polymer melts via nonequilibrium molecular dynamics, J. Rheol. 37 (1993) 1057.

[40] S. Heidenreich, P. Ilg, S. Hess, Robustness of the periodic and chaotic orientational behavior of tumbling nematic liquid crystals, Phys. Rev. E 73 (2006) 061710.

[41] P. Ilg, I.V. Karlin, H.C. Öttinger, Generating moment equations in the Doi model of liquid-crystalline polymers, Phys. Rev. E 60 (1999) 5783

[42] P. Ilg, M. Kröger, S. Hess, Magnetoviscosity and orientational order parameters of dilute ferrofluids, J. Chem. Phys. 116 (2002) 9078.

[43] P.G. de Gennes, The Physics of Liquid Crystals, Oxford Univ. Press, Oxford, 1974.

[44] T. Erdmann, M. Kröger, S. Hess, Phase behavior and structure of Janus fluids, Phys. Rev. E 67 (2003) 041209.

[45] M. Kröger, P. Ilg, Derivation of Frank-Ericksen elastic coefficients for polydomain nematics from mean-field molecular theory for anisotropic particles, J. Chem. Phys. 127 (2007) 034903.

[46] V. Faraoni, M. Grosso, S. Crescitelli, P.L. Maffettone, The rigid-rod model for nematic polymers: an analysis of the shear flow problem, J. Rheol. 43 (1999) 829.

[47] A. Ammar, D. Ryckelynck, F. Chinesta, R. Keunings, On the reduction of kinetic theory models related to finitely extensible dumbbells, J. NonNewtonian Fluid Mech. 134 (2006) 136-147.

[48] J. Herzfeld, A.E. Berger, J.W. Wingate, A highly convergent algorithm for computing the orientation distribution functions of rodlike particles, Macromolecules 17 (1984) 1718 